\title{
Provider Performance Reports and Consumer Welfare
}

\author{
Henry Y. Mak \\ Indiana University-Purdue University Indianapolis \\ makh@iupui.edu
}

August 2016

\begin{abstract}
A provider's performance report consists of his service average outcome and volume. The two variables depend on the provider's private quality type and current demand, but he can raise his average outcome by dumping vulnerable consumers. Prospective consumers infer providers' qualities from their reports. Performance reporting drives some providers to dump consumers when competition is intense, but it may not reveal providers' qualities when their average quality is high. Statistical adjustment aiming at making reports independent of consumer characteristics can lead to more dumping, less informative reports, or both. There is more dumping when volume information is withheld and less dumping when ratings information is coarse.
\end{abstract}

Acknowledgement: This article is based on a chapter of my Ph.D. dissertation at Boston University. I am indebted to Ching-to Albert Ma for his guidance and support. I also wish to thank Sambuddha Ghosh, Jacob Glazer, Michael Luca, and participants at various seminars and conferences for their comments. I am grateful to the coeditor Kathryn Spier and two anonymous referees for their suggestions. All errors are my own.

This is the author's manuscript of the article published in final edited form as:

Mak, H. Y. (2017). Provider performance reports and consumer welfare. The RAND Journal of Economics, 1(48), $250-280$.

http://dx.doi.org/10.1111/1756-2171.12175 


\section{Introduction}

"Success should be judged by results, and data is a powerful tool to determine results. We can't ignore facts. We can't ignore data."

President Barack Obama, July 24, 2009

Public reporting is instituted to collect and disseminate performance data so that success can be judged. New York State started reporting cardiac surgery performance in 1989. Since then, public performance reporting has penetrated the health care sector and other sectors such as social services, crime and enforcement, and education, in the United States and elsewhere.

Unlike private agencies that can report opaque or subjective performance measures, public agencies are often obligated to base their reports on transparent and objective measures. ${ }^{1}$ Therefore, most public performance reports use end results as measures of underlying service qualities. Because results often depend on clientele, some public performance reports are adjusted to isolate service qualities from consumer characteristics. Table 1 gives some examples of public performance reports under four categories based on their contents. $^{2}$

\begin{tabular}{ll} 
Contents of reports & Examples \\
\hline 1. Average outcome and volume & $\begin{array}{l}\text { Child protection investigation completion } \\
\text { rate and number of cases of a district } \\
\text { 2urgery mortality rate of a hospital adjusted } \\
\text { for patient severities and number of cases }\end{array}$ \\
& $\begin{array}{l}\text { Standardized test passing rate of a school } \\
\text { Job placement rate of a training center } \\
\text { 3. Average outcome }\end{array}$ \\
4. Adjusted for enrollment demographics
\end{tabular}

Table 1: Four categories of public performance reports

Although these reports have different contents, they share a common purpose of using results to direct consumer choice and resource allocation. For example, surgery outcome reports induce patients to switch

\footnotetext{
${ }^{1}$ For example, Google search uses secret algorithms to rank the relevance of web pages. Ederer et al. (2014) show that opaque incentive schemes can outperform transparent ones when agents are strategic. A large literature studies subjective performance evaluation; see, for example, Levin (2003) and MacLeod (2003).

${ }^{2}$ These examples are from Florida Department of Children and Families (http://dcfdashboard.dcf.state.fl.us); New York Department of Health (http://health.ny.gov/statistics); Indiana Department of Education (http://compass.doe.in.gov); and United States Job Training Partnership Act (JTPA) program (http://wdr.doleta.gov/opr/fulltext/99-performance.pdf). The JTPA program was replaced by the Workforce Investment Act program in 2000.
} 
from high-mortality to low-mortality hospitals; job placement reports allow funding authorities to determine training centers' budgets according to their placement rates. These changes benefit patients and trainees if the end results are perfect measures of service qualities.

However, a provider is likely to have more information about his consumers than the public reporting agency. This allows a provider to manipulate his end results strategically. For example, a low quality school can raise its standardized test passing rate by retaining weaker students and increasing special education placements (Jacob, 2005). These strategic behaviors can harm the affected students, and defeat the purpose of performance reporting by making the passing rates uninformative.

In this article, I develop a model to study how public performance reporting affects providers' service decisions and consumer welfare. Providers in the model have private information about their service qualities and demands. They can manipulate their reports by dumping consumers with certain characteristics. ${ }^{3,4}$ The model shows how the four categories of reports in Table 1 yield different dumping choices, report informativeness, and consumer welfare. In particular, I show that reporting adjusted outcomes (categories 2 and 4 in Table 1) may lead to more dumping, less informative reports, and lower consumer welfare. These results can account for a number of empirical findings. They are also useful for the design of public performance reporting strategy.

For concreteness, consider a market for surgery as an application. In each of two periods, some new patients would like to receive treatments from one of two providers. Each patient's severity can be high or low and each provider's quality type can be good or bad. A patient's treatment outcome depends on her severity and the quality type of her provider. The number of patients who request a provider's service is stochastic, but increasing in the provider's perceived quality. Each provider receives positive payoffs from providing services.

A provider's quality type is his private information. If there is no performance reporting, the demands

\footnotetext{
${ }^{3}$ In this article, I follow Ellis (1997) and use the term dumping to refer the avoidance of consumers with worse service outcomes. However, many authors use the terms dumping and cream-skimming interchangeably.

${ }^{4}$ In addition to dumping weaker students, Dranove et al. (2003) find that cardiac surgery performance reports have led surgeons to dump sicker patients; Heckman and Smith (2004) find that applicants with less education and from poorer families are less likely to be enrolled for job training programs.
} 
in both periods are independent of the providers' quality types. Now suppose a public reporting agency observes and reports the quality type of each provider at the end of period one. These reports induce some patients in period two to switch from a bad provider to a good provider. ${ }^{5}$ Because the providers' reports are independent of their treatment choices, they have no incentives to dump patients in either period.

Next, suppose the reporting agency can only observe and report each provider's average treatment outcome and volume (report category 1 in Table 1 ) at the end of period one. Now a provider's report depends on his quality type as well as the number of patients who request his services in period one, and whom among those patients he chooses to treat. If a good provider receives many treatment requests in period one, the provider can obtain a report with high average treatment outcome and high volume by serving all his patients. This report will convince the patients in period two that the provider is a good type, as if the agency observed and reported the provider's type.

However, a good provider who receives fewer treatment requests cannot reveal his type by treating all his patients in period one. This is because his report can be mimicked by a bad provider who receives many requests in period one and dumps some of his patients. Dumping is costly to both provider types in question. However, patient reactions to performance reports in period two create incentive trade-offs between treating more patients in period one, and getting more patients in period two. These trade-offs shape the equilibrium outcomes.

In partial pooling equilibria, both a good provider with fewer requests and a bad provider with many requests have the same reported average outcome and volume. Partial pooling equilibria are more likely to exist when patients are responsive to quality difference and the providers' expected quality is high, so that a perceived bad type loses a significant amount of patients in period two. Comparing this with the benchmark case where the agency observes quality types, there are two sources of inefficiency. First, at least one provider type who obtains the partial pooling report dumps patients in period one. ${ }^{6}$ Second, the equilibrium reports

\footnotetext{
${ }^{5}$ Although the model focuses on consumer response, it can be easily modified to accommodate explicit rewards and sanctions for performance.

${ }^{6}$ It is not possible for a bad provider with fewer treatment requests in period one to mimic any good provider under report category 1 . Hence, this provider must accept all patients in period one and reveal his type in any equilibrium.
} 
are less informative to the patients in period two. These inefficiencies can make performance reporting welfare-deteriorating. Furthermore, the partial pooling equilibrium with the least dumping cost is selected by the undefeated equilibrium refinement (Mailath et al., 1993) as the game's unique equilibrium when the providers' expected quality is sufficiently high.

However, the separating equilibria with the least dumping costs are the only undefeated equilibria when patients are not responsive to quality difference, or when patients are responsive but the providers' expected quality is low. Separating performance reports fully reveal each provider's quality type, and hence there is no inefficiency in period two. Yet, a good provider with fewer requests can treat all his patients in period one only if patients are not responsive to quality difference. Otherwise, the good provider has to dump some of his sicker patients to raise his average outcome and credibly separate from a bad type with many requests. This inefficiency alone can make the separating reports welfare-deteriorating too.

Now let the agency switch to reporting adjusted outcomes and volumes (report category 2). Furthermore, suppose the reporting agency cannot observe a patient's severity but it can observe the patient's age, and age is an informative signal in the sense that older patients are more likely to be sicker. The agency can adjust the average treatment outcomes by comparing the patients' actual treatment outcomes with the expected treatment outcomes based on the patients' ages. ${ }^{7}$ Because severity and age are correlated, a sicker patient's poor treatment outcome is compensated by a poor expected treatment outcome. Dumping sicker patients would become a less effective way to manipulate performance reports.

I show that this logic fails when severity and age are not highly correlated. This is because statistical adjustment alters the information asymmetry problem in a subtle way. Although the reporting agency can infer a patient's expected severity from her age, a provider knows his patient's severity better. When severity and age are not highly correlated, a small but significant proportion of sicker patients are young. The agency expects them to have good treatment outcomes. By dumping these patients, a bad provider with many treatment requests can raise his adjusted average more than his unadjusted average. It becomes easier for a bad provider to mimic or pool with a good provider with fewer requests.

\footnotetext{
${ }^{7}$ This kind of statistical adjustment is termed risk adjustment in the health care sector and value-added modelling in the education sector.
} 
When the agency ceases to report volumes, report categories 1 and 2 in Table 1 become categories 3 and 4 , respectively. ${ }^{8}$ It is perhaps not surprising that reports without volume information are more prone to gaming. However, I find that adjusting the average outcomes can still lead to more dumping when volumes are not reported. Finally, I show that in my environment a coarse reporting scheme can eliminate all dumping and reveal some quality information. This reporting scheme can improve consumer welfare over report categories 1 and 2 when the efficiency loss from dumping is severe.

\section{Evidence and literature}

My model offers a systematic way to interpret empirical evidence on dumping, information content of performance reports, and consumer welfare. Take the cardiac surgery outcome reporting initiative for example. Zhang (2011) shows that the introduction of surgeon performance reports in New York have led to gaming in Manhattan, where competition is fierce, but not elsewhere. This observation is consistent with the prediction of my model.

My model also predicts that separating performance reports can make consumers worse off because of dumping by good providers. In New York, informative reports have improved matching between hospitals and providers, but worsened patient outcomes (Dranove et al., 2003). Moreover, Jha and Epstein (2006) find that surgeons with good reports left New York practices because of "the pressure to reject high-risk patients and focus on documentation".

On the other hand, in Massachusetts, the first three annual cardiac surgery outcome reports show no significant performance difference between hospitals in the state (Mass-DAC, 2005-07). Yet, the proportion of high-risk patients undergoing heart surgeries in Massachusetts has declined by $47 \%$ in the same period (Resnic and Welt, 2009). My model suggests that such equilibrium outcome can exist when the providers are likely to be good.

I show that adjusting outcomes with inaccurate information about consumer characteristics cannot reduce gaming. This result is of practical relevance. Using data from a large U.S. federal job training program (see

\footnotetext{
${ }^{8}$ An alternative interpretation is that consumers fail to understand the informational content of a provider's service volume.
} 
the JTPA program in footnote 2), Courty et al. (2011) find that training centers improve their adjusted placement outcomes by selecting enrollees according to characteristics missed by the adjustment model. Recently, many reporting agencies in the education sector use adjusted student test scores to evaluate schools and teachers. These agencies use past test scores to control for student characteristics. However, using data from North Carolina, Kane and Staiger (2002) find that only $60 \%$ of the variations in test-score gains were persistent over time.

In the health care sector, Brown et al. (2014) find that adjusting Medicare payments by enrollee risk factors has led to insurers' gaming and higher government expenditure. Similar statistical models are used to adjust provider performance reports. However, providers are likely to have more information about their patients than insurers do. For example, $44.7 \%$ of patients in Massachusetts who died after cardiac surgery at Brigham and Women's Hospital had at least one severe medical condition not considered by the reporting agency's risk adjustment model (Resnic and Welt, 2009). ${ }^{9}$

The literature on quality disclosure is large. Grossman (1981) and Milgrom (1981) show that demand response is sufficient to drive providers to reveal their private quality information voluntarily. Many subsequent articles examine the underlying assumptions of this unraveling result, and recommend mandatory quality reporting when those assumptions are not met. ${ }^{10}$ However, these articles are based on a common assumption that quality information is private but perfectly verifiable. ${ }^{11}$

Quality information is not perfectly verifiable in my model as well as in Holmstrom (1999). In Holmstrom's career concern model, a single provider's observable performance depends on his ability type, his private action, and a random shock. The provider's type is commonly unknown, and he exerts type-independent effort to influence market perception about his ability type. I introduce provider competition in a way similar

\footnotetext{
${ }^{9} \mathrm{I}$ will further discuss empirical evidence that is consistent with other testable predictions of the model in Section 7.

${ }^{10}$ Section 2.1 of Dranove and Jin (2010) offers a comprehensive review of theoretical research on voluntary and mandatory quality disclosure.

${ }^{11}$ In Matthews and Postlewaite (1985) and Bar-Issac et al. (2012), mandatory disclosure can be welfaredeteriorating because a provider may refrain from acquiring quality information or underprovide unreported qualities. On the other hand, Lizzeri (1999), Albano and Lizzeri (2001) focus on profit-maximizing reporting agencies' incentives to manipulate quality reports.
} 
to Daughety and Reinganum (2007) to make demands in period one independent of the providers' dumping decisions. This allows a provider's quality type and demand shock to be his private information, and his dumping decisions to be type and demand dependent.

Under report categories 1 and 2 in Table 1, consumers in period two infer a provider's private quality type from both his unadjusted/adjusted average outcome and volume. This is essentially a multidimensional signalling problem. The literature on multidimensional signalling is small. Chen (1997) studies a game where a firm has private information about cost and demand (both binary). The firm can use diversification and financing activities (again both binary) to signal its private information. Chen characterizes separating equilibria and two kinds of partial pooling equilibria. Bagwell (2007) considers an entry deterrence game in which the incumbent has private information about his cost and patience types (both binary). The incumbent can use any nonnegative price and advertising level to signal its types, but the entrant is interested in the incumbent's cost type only. Bagwell shows that three kinds of partial pooling equilibria can survive the intuitive criterion (Cho and Kreps, 1987).

In line with Chen (1997) and Bagwell (2007), I assume a provider has private information about his quality type and period-one demand, and they are both binary. However, the report content (unadjusted/adjusted average outcome and volume) through which a provider signals his quality type to period-two consumers is controlled by the reporting agency. This allows me to compare providers' strategic responses under the four categories of reports in the multidimensional signalling framework. I show how the report content determines the existence of a unique kind of refined partial pooling equilibria, and the welfare properties of refined separating equilibria. ${ }^{12}$

Chen (2011) also considers performance reporting as a multidimensional signalling problem. He focuses on report category 1. However, providers' demands are deterministic and their quality types are perfectly correlated in his model. Therefore, it is impossible for a bad provider to mimic a good provider when each provider faces an identical distribution of patients. He then allows an informed physician to refer more

\footnotetext{
${ }^{12}$ Fong (2009) derives optimal screening contracts that separate a good provider from a bad provider. In Ely and Valimaki (2003) the strategic behavior of a good provider causes consumers to refuse to hire, hence the market breaks down.
} 
patients who are sicker to the good provider. The uneven distributions of patients lead the good provider to dump patients.

Glazer and McGuire (2000) is the first article to study statistical adjustment in an equilibrium model. They show that using informative signals to adjust insurance premiums statistically can mitigate adverse selection in the Rothschild and Stiglitz (1976) screening framework. They further show that adverse selection can be completely eliminated by overpaying the bad signal and underpaying the good signal. So far this literature has exclusively focused on screening models. In this article I consider signalling and show that adjusting performance reports statistically can intensify rather than mitigate patient selection. ${ }^{13,} 14$

The rest of the article is organized as follows. Section 2 sets up a baseline model where a provider's performance report is about service volume and average outcome. Section 3 introduces two informational benchmarks, and considers the feasibility and cost of report manipulation. Section 4 characterizes two classes of equilibria, which are further analyzed and compared to the benchmarks in Section 5. Section 6 considers the effects of statistically adjusting performance reports. Section 7 discusses some of the model's testable predictions and extensions, and then concludes the article. The Appendix contains all proofs omitted in the main text. Finally, the Supplement extends the model by allowing prices to vary with providers' perceived service qualities, and considers the refinement of the equilibria in Section 4 by the intuitive criterion.

\section{$2 \quad$ Model}

\section{Providers, consumers, and services}

Consider two service providers, Provider $x$ and Provider $y$. Each provider $i=x, y$ belongs to one of two quality types: bad or good. Provider $i$ 's quality type is captured by a variable $T_{i} \in\{B, G\}$, where $B<G$ and $B$ is normalized to zero. There are many consumers. Each consumer's characteristic is captured by a

\footnotetext{
${ }^{13} \mathrm{Ma}$ and Mak (2014) consider a monopolist who uses a menu of multi-quality goods to screen consumers. The monopolist's quality choices are observed by a reporting agency only. The article shows that the agency can mitigate the monopolist's quality distortion by reporting just the average quality of each good.

${ }^{14} \mathrm{Ma}$ and Mak (2015) show that by bundling quality and cost information optimally, a regulator can induce a provider to invest in cost efficiency even if its cost is fully reimbursed. I will briefly consider the benefit and cost of coarsening performance reports in Section 7.
} 
binary variable $s \in\{l, h\}$ (low and high), where $0<l<h$. I will sometimes use the terms "a $T_{i}$ type" and "an $s$ consumer".

Each consumer demands one unit of service. Each performed service generates an outcome. If a $T_{i}$ type serves an $s$ consumer, the outcome is $T_{i}+s$. This simple formulation captures how the provider quality type and consumer characteristic affect service outcomes. First, for a given consumer characteristic $s$, service outcome is better if the consumer is served by a good type, and the parameter $G$ can be interpreted as a service quality premium. Second, for a given provider quality type $T_{i}$, an $h$ consumer has a better service outcome than an $l$ consumer.

A consumer's payoff from service also depends on her own characteristic and her provider's quality type. An $s$ consumer receives $U_{T}^{s}=T_{i}+v^{s}-P$ from a $T_{i}$ type's service. Because $B<G$, a consumer benefits more from a $G$ type's service. The variable $v^{s}$ captures how a consumer's characteristic affects her payoff from service. I let $0<v^{h} \leq v^{l}$, that is, an $l$ consumer receives a weakly higher payoff than an $h$ consumer if both are served by the same type of provider. The positive parameter $P$ is the service price and $P<v^{h}$, hence $U_{T}^{s}$ is always strictly positive. ${ }^{15}$

The distinction between outcome and payoff may first appear to be unnatural. I now use some examples in Table 1 to illustrate the difference. In the surgery report example, a consumer's characteristic is about her health status. A surgery outcome is a measure such as 30-day mortality rate or readmission rate. A sicker patient is more likely to die or be readmitted after surgery, but may benefit more from surgery over alternative treatments. In the job training example, a consumer's characteristic is about her initial employment readiness. A training outcome is some short-term measures such as hourly wage and employment rate upon completion. A trainee's payoff is about her long-term earnings and employment gain. Heckman et al. (2002) find that these short- and long-term measures are either uncorrelated or negatively correlated. ${ }^{16}$

This article is about how public performance reports distort service-provision decisions in both public and private sectors. In the model, I allow providers to receive both intrinsic and extrinsic rewards from

\footnotetext{
${ }^{15}$ I use $G$ to denote quality premium in terms of both outcome and payoff for ease in notation. This can be considered as normalizing one of the two dimensions.

${ }^{16}$ I will further consider the implication of $v^{l}<v^{h}$ in Section 5.
} 
service provisions. When a $T_{i}$ type serves an $s$ consumer, the provider's payoff is $\prod_{T}^{s}=\omega\left(T_{i}+v^{s}\right)+(1-\omega) P$, where $0 \leq \omega \leq 1 .{ }^{17}$ Hence, $\Pi_{T}^{s}$ is a weighted sum of the $s$ consumer's net payoff and the $T_{i}$ type's profit, where the service cost is set to zero for simplicity.

\section{Information, demand response, and dumping}

The providers live two periods. A provider's quality type is his private information. Each provider is a good type with probability $\alpha$, and a bad type with probability $1-\alpha$. The providers are ex ante identical. Their types are drawn independently at the beginning of the game and fixed over the two periods. A consumer lives in either period one or two. A consumer's characteristic is $h$ with probability $\lambda$, and $l$ with probability $1-\lambda$. Each consumer's characteristic is drawn independently, and the realization is only observed by herself and the provider she visits.

A provider's demand is the mass of atomless consumers who request his services in a period. The two providers' demands are determined in each period by both the state of nature and consumers' beliefs about the providers' quality types. First, suppose the consumers perceive that the two providers are of the same quality type. Provider $i$ 's demand in this case is $q_{i} \in\{1, k\}$, where $1<k$. That is, the mass of consumers in the low-demand state is normalized to one. The draws of $q_{i}$ are independent and $q_{i}=k$ with probability $\beta$. A provider's expected demand in this case is $\widehat{q} \equiv \beta k+(1-\beta)$.

Now suppose the consumers perceive that the quality types of Providers $x$ and $y$ are respectively $\widetilde{T}_{x}$ and $\widetilde{T}_{y}$, where $\widetilde{T}_{x}<\widetilde{T}_{y}$. Here, I let $\tau\left(\widetilde{T}_{y}-\widetilde{T}_{x}\right) q_{x}$ be the mass of Provider $x$ 's consumers who switch to Provider $y$. The positive parameter $\tau$ is a measure of demand responsiveness to perceived quality differences. ${ }^{18}$ The expected demands of Providers $x$ and $y$ in this case are $\left[1-\tau\left(\widetilde{T}_{y}-\widetilde{T}_{x}\right)\right] \widehat{q}$ and $\left[1+\tau\left(\widetilde{T}_{y}-\widetilde{T}_{x}\right)\right] \widehat{q}$, respectively. ${ }^{19}$ I assume $0<\tau<\bar{\tau}$ and $\bar{\tau}<\frac{1}{G}$, so that $\tau\left(\widetilde{T}_{y}-\widetilde{T}_{x}\right)$ is strictly less than one. The case where $\widetilde{T}_{y}<\widetilde{T}_{x}$ is

\footnotetext{
${ }^{17}$ See, for example, Francois (2000) on public service motivation and McGuire (2000) on physician altruism. The intrinsic rewards are in the form of warm-glow payoffs or impure altruism (Andreoni, 1989). I will also consider the implication of assuming providers to be purely altruistic in Footnote 36.

${ }^{18}$ All the results in this article hold qualitatively if the demand responsiveness of $l$ and $h$ consumers are $\tau^{l}$ and $\tau^{h}$, respectively, and $\tau^{l} \neq \tau^{h}$.

${ }^{19}$ Note that these demand functions are identical to those derived from a Hotelling model with linear transportation costs when $q_{i}$ are deterministic.
} 
analogous.

Consumers in both periods cannot directly observe the providers' quality types or demands. However, a non-strategic reporting agency disseminates performance reports of the two providers at the beginning of period two. Provider $i$ 's report, $\left(M_{i}, A_{i}\right)$, is about the mass of consumers he has served in period one, $M_{i}$, and the average service outcome of these consumers, $A_{i}$. These numbers depend on the provider's demand, quality type, and the characteristic of the consumers that the provider chooses to serve in period one (see Section 3). In particular, a provider observes his consumers' types and he can dump some of his consumers based on consumer characteristic.

As discussed in the introduction, consumer dumping can take many forms and is difficult to monitor in practice. In the model, I assume that the reporting agency cannot observe the demand or mass of consumers that a provider dumps in period one. Moreover, the reporting agency cannot observe the characteristic of consumers whom a provider chooses to serve. I will allow the agency to have some information about the consumers' characteristic in Section 6.

Dumping is costly for both providers and consumers. When a provider dumps his consumer, the payoffs of both parties become zero. Hence, a $T_{i}$ type and an $s$ consumer lose, respectively, $\Pi_{T}^{s}$ and $U_{T}^{s}$ from dumping. A provider's objective is to maximize his aggregate payoff over the two periods. There is no discounting between periods.

\section{Extensive form and solution concept}

The extensive form of the game is as follows.

Stage 1A: Nature decides the two providers' quality types and privately informs each provider of his own type. Nature together with consumers' beliefs determine the two providers' period-one demands.

Stage 1B: Each provider observes his period-one demand. He chooses to serve or dump each of his consumers after observing their characteristic. The services are performed and period one ends.

Stage 2A: The reporting agency announces the period-one performance reports. Nature together with consumers' beliefs determine the two providers' period-two demands. 
Stage 2B: Each provider observes his period-two demand. He chooses to serve or dump each of his consumers after observing their characteristic. The services are performed and the game ends.

I characterize pure strategy perfect Bayesian equilibria of the game. Because the prior distributions over the quality types $T_{i}$ and exogenous demands $q_{i}$ are the same for Providers $x$ and $y$, I will focus on symmetric equilibria. An equilibrium is symmetric if the two providers use the same strategy and the consumers' inferences about a provider's quality type depend only on his report but not his identity $i$.

\section{Preliminary analysis}

\section{Benchmarks: Perfect Report and No Report}

I first introduce two benchmarks. Under Perfect Report, the providers' quality types are perfectly verifiable as in the quality disclosure literature. The reporting agency observes and reports the quality types, $\left(T_{x}, T_{y}\right)$, of the two providers in Stage 2A. ${ }^{20}$ A provider's report is independent of the realization of his demand and service decisions in period one. Because $\Pi_{T}^{s}$ is strictly positive, the two providers serve all their consumers in both periods. However, the benchmark preserves information asymmetry in period one. In this period, the uninformed consumers must perceive that the two providers offer services with the same expected quality. Therefore, each provider's expected demand is $\widehat{q}$, irrespective of his quality type. The consumers in period two can respond to the perfect reports. The expected demands of the two providers are:

\begin{tabular}{|c|c|c|c|c|}
\hline$T_{x}, T_{y}$ & $G, G$ & $G, B$ & $B, G$ & $B, B$ \\
\hline Provider $x$ 's expected demand & $\widehat{q}$ & $(1+\tau G) \widehat{q}$ & $(1-\tau G) \widehat{q}$ & $\widehat{q}$ \\
\hline Provider $y$ 's expected demand & $\widehat{q}$ & $(1-\tau G) \widehat{q}$ & $(1+\tau G) \widehat{q}$ & $\widehat{q}$ \\
\hline
\end{tabular}

Table 2: Expected demands in period two under Perfect Report

The second benchmark is No Report. If there are no performance reports, the providers cannot convey credible information about their quality types in either period. Each provider's expected demand is $\widehat{q}$ and he serves all his consumers in both periods, irrespective of his quality type.

\footnotetext{
${ }^{20}$ This benchmark was first introduced by Mailath (1989) to analyze the welfare properties of simultaneous signalling. The case where consumers in every period know the quality type of each provider cannot be achieved by reports on past performances, and hence is a less relevant benchmark.
} 


\section{Feasible performance reports}

Now consider the extensive-form game in Section 2. In this game, the providers' expected demands in Stage $1 \mathrm{~A}$ and their service decisions in Stage 2B are identical to those in the two benchmarks. In Stage 1A, quality information is not available. Consumers must perceive the providers as identical. In Stage 2B, the last stage of the game, the positive payoffs $\Pi_{T}^{s}$ drive the providers to serve all consumers.

However, the two providers' service decisions in Stage 1B and their expected demands in Stage 2A are linked by performance reporting. In Stage 1B, Provider $i$ 's service decisions influence his report $\left(M_{i}, A_{i}\right)$. The report then influences consumers' beliefs and hence the provider's expected demand in Stage 2A. This demand response, in turn, influences the provider's service decisions in Stage 1B.

I first consider the linkage between a provider's service decisions in Stage 1B and his report. Because a provider's choice is independent of his identity $i$ in a symmetric equilibrium, I will remove the subscript $i$ when there is no confusion. Moreover, I will use the term "a $T q$ provider" to summarize a provider's private information in Stage 1B, that is, his quality type $T$ and his period-one demand $q$. Now suppose a $T q$ provider dumps $D_{T q}^{s}$ of his $s$ consumers in Stage 1B. His performance report is

$$
(M, A)=\left(q-D_{T q}^{h}-D_{T q}^{l}, \frac{\left\{q \lambda-D_{T q}^{h}\right\}(T+h)+\left\{q(1-\lambda)-D_{T q}^{l}\right\}(T+l)}{q-D_{T q}^{h}-D_{T q}^{l}}\right) .
$$

Invert equation (1), a $T q$ provider must dump

$$
\left(D_{T q}^{h}(M, A), D_{T q}^{l}(M, A)\right)=\left(q \lambda-\left(\frac{A-T-l}{h-l}\right) M, q(1-\lambda)-\left(\frac{T+h-A}{h-l}\right) M\right)
$$

of his $h$ and $l$ consumers to obtain report $(M, A)$, where $M \in[0, q]$ and $A \in[T+l, T+h] \cup 0$.

The left panel of Figure 1 demonstrates the relationship among a provider's quality type, demand and service decisions in period one, and his report. First consider point $a$ where $M=1$ and $A=G+\widehat{s}$, $\widehat{s} \equiv \lambda h+(1-\lambda) l$. This is the report a $G 1$ provider obtains if he serves all his consumers in period one. Starting from this point, the provider can raise his average outcome $A$ by dumping some $l$ consumers, or lower his $A$ by dumping some $h$ consumers. However, dumping will lower the mass of served consumers $M$. The area bounded by the solid curves represents the combinations of $(M, A)$ that the provider can obtain by dumping consumers. Points $b, c$, and $d$ in the diagram respectively represent the reports that $G k, B 1$, 

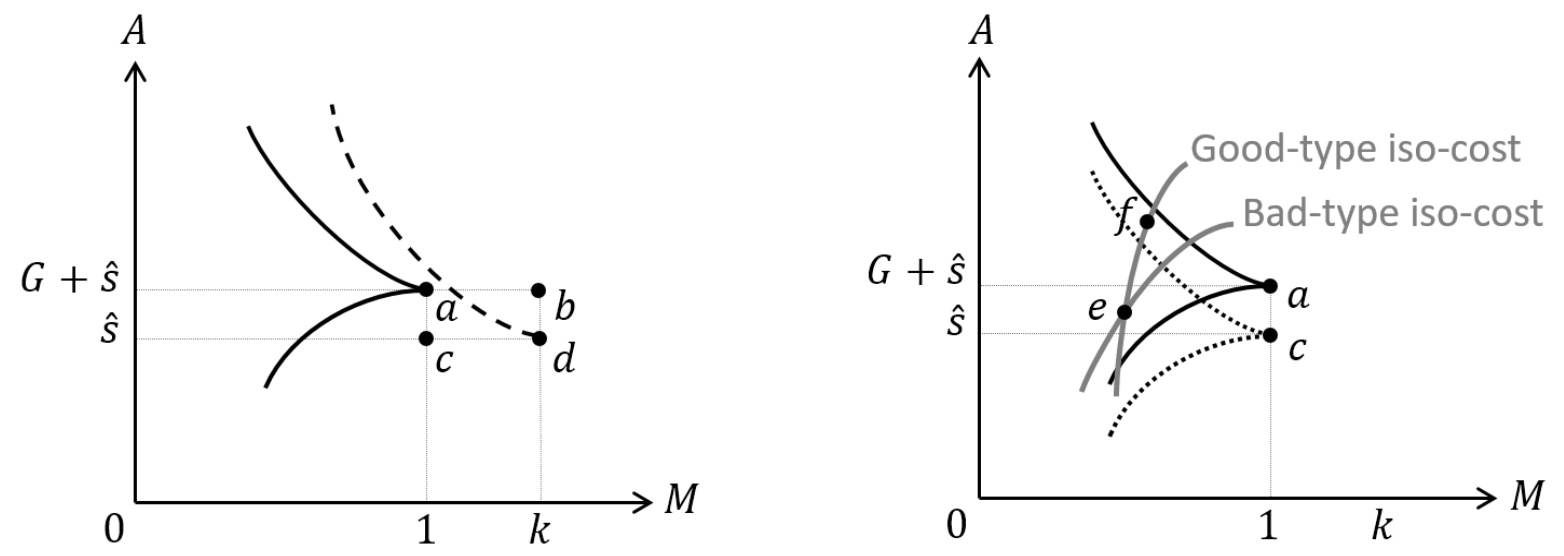

Figure 1: Feasibility of gaming and iso-cost curves

and $B k$ providers obtain if they serve all consumers in period one.

Because of the demand responses in period two, a bad type wants to be perceived as a good type irrespective of his demand in period one. However, because a $G k$ provider has both quality premium and high demand, it is impossible for $B 1$ and $B k$ providers to mimic his report, even if the $G k$ provider serves all his consumers in period one. How about mimicking a $G 1$ provider? The quality difference prevents a $B 1$ provider from mimicking a $G 1$ provider who dumps no consumers. However, the following lemma provides conditions such that a $B k$ provider can mimic a $G 1$ provider who serves all his consumers.

Lemma 1 The performance report of a Gk provider who serves all his consumers cannot be mimicked. However, assume that $k-1<k(1-\lambda)$, the report of a $G 1$ provider who serves all his consumers can be mimicked by a Bk provider who dumps some of his consumers if

$$
G \leq(k-1) \lambda(h-l)
$$

The inequality $k-1<k(1-\lambda)$ says that a $B k$ provider's demand from $l$ consumers, $k(1-\lambda)$, is sufficiently big, so that he can achieve $M=1$ by dumping some but not all of his $l$ consumers. The inequality in (3) says that the quality premium $G$ is sufficiently small, so that a $B k$ provider can achieve $A \geq G+\widehat{s}$ by dumping $k-1$ of his $l$ consumers. The two inequalities imply that point $a$ in the left panel of Figure 1 is to the 
southwest of the downward-sloping dashed curve. ${ }^{21,22}$

\section{Iso-dumping-cost curves}

Lemma 1 establishes the feasibility of gaming the performance reports. I now turn to the providers' dumping costs in Stage 1B. Because a $T q$ provider loses $\Pi_{T}^{s}$ from dumping an $s$ consumer, the provider's dumping cost is the same for any combinations of $D_{T q}^{h}$ and $D_{T q}^{l}$ that satisfy $D_{T q}^{h} \Pi_{T}^{h}+D_{T q}^{l} \Pi_{T}^{l}=F$, where $F$ is a positive constant. Using the definitions of $D_{T q}^{h}(M, A)$ and $D_{T q}^{l}(M, A)$ in (2), I can define a $T q$ provider's iso-dumping-cost curve in terms of $M$ and $A$. Totally differentiating the equation with respect to $M$ and $A$, I obtain the slopes of the iso-cost curves

$$
\frac{d A}{d M}=\frac{\Pi_{T}^{l}(T+h-A)+\Pi_{T}^{h}(A-T-l)}{M\left(\Pi_{T}^{l}-\Pi_{T}^{h}\right)},
$$

which is positive and independent of demand realization $q$.

The right panel of Figure 1 depicts two iso-cost curves of bad and good types, respectively. The upwardsloping and convex iso-cost curves illustrate the case where $\Pi_{T}^{l}-\Pi_{T}^{h}=\omega\left(v^{l}-v^{h}\right)>0$. A provider raises his average outcome $A$ by dumping more $l$ consumers. When $\omega\left(v^{l}-v^{h}\right)>0$, it is more costly for both $G$ and $B$ types to dump $l$ consumers. A provider has to dump less consumers when he raises $A$ along an iso-cost curve. In addition, a $G$ type's iso-cost curve is steeper than that of a $B$ type everywhere when $\omega\left(v^{l}-v^{h}\right)>0$. This is because the quality premium $G$ reduces a good type's relative cost of dumping an $l$ consumer $\left(\frac{\Pi_{G}^{l}}{\Pi_{G}^{h}}<\frac{\Pi_{B}^{l}}{\Pi_{B}^{h}}\right) \cdot{ }^{23}$

When $\omega\left(v^{l}-v^{h}\right)=0$, a provider's costs of dumping $h$ and $l$ consumers are identical. The iso-cost curves of both bad and good types become vertical lines. When I analyze this case in the following sections, I will

\footnotetext{
${ }^{21}$ Suppose the proportions of $l$ consumers are different when a provider's period-one demand is 1 and $k$, so that points $b$ and $d$ in the panel shift either up or down. The qualitative results in this article continue to hold as long as Lemma 1 is satisfied.

${ }^{22}$ The case where a provider has private information about his quality type, his demand, and the distribution of his consumers' characteristics is beyond the scope of this article. However, I will consider the implications of provider-consumer matching in Section 7.

${ }^{23}$ Let $G^{h} \neq G^{l}$, a good type has a lower relative cost of dumping $l$ consumers if $\frac{G^{l}}{G^{h}}<\frac{\Pi_{B}^{l}}{\Pi_{B}^{h}}$. Because $\Pi_{B}^{l}-\Pi_{B}^{h}=$ $\omega\left(v^{l}-v^{h}\right) \geq 0$, a good type can have a lower relative cost of dumping $l$ consumers even if he has a comparative advantage in serving $l$ consumers $\left(G^{h}<G^{l}\right)$.
} 
impose a tie-breaking rule by which a provider who is indifferent between dumping an $h$ and an $l$ consumer will dump the $l$ consumer. Finally, the slopes of the iso-cost curves have an immediate implication.

Lemma 2 There is no equilibrium in which a B1 provider and a $G 1$ provider obtain identical performance reports.

To see why this lemma is true, consider the right panel of Figure 1 again. Suppose point $e$ represents the equilibrium reports of both $B 1$ and $G 1$ providers. In period two, consumers must have the same perceptions about their quality types. Now if the $G 1$ provider moves to point $f$ on his Good-type iso-cost curve, his dumping cost remains unchanged, but the new report is infeasible for the $B 1$ provider to obtain. Hence the $G 1$ provider can raise his expected demand in period two by deviating from point $e$ to point $f$, which is a contradiction.

Lemmas 1 and 2 imply that both $B 1$ and $G k$ providers cannot pool with other providers, and hence they must serve all their consumers in any equilibrium. I will next consider the inter-period trade-offs of $B k$ and G1 providers and characterize the equilibrium outcomes.

\section{Equilibrium performance report and dumping}

There are two classes of equilibria. In a partial pooling equilibrium, both $B k$ and $G 1$ providers obtain identical performance reports. In a separating equilibrium, a provider's quality type is completely revealed. I first study partial pooling equilibria.

\section{Partial pooling equilibria}

In a partial pooling equilibrium, $B 1$ and $G k$ providers obtain distinctive reports, but $B k$ and $G 1$ providers obtain a common level of report $\left(M_{p}, A_{p}\right)$. Consumers in period two cannot ascertain a provider's quality type from $\left(M_{p}, A_{p}\right)$. Because of the differences in quality and demand between $B k$ and $G 1$ providers, at least one of them dumps consumers in period one.

I first characterize equilibria with the consumers' beliefs that a provider with any off-equilibrium report is a bad type. Given the beliefs, the following two incentive constraints summarize the inter-period incentive 
trade-offs of $B k$ and $G 1$ providers, respectively:

$$
\begin{aligned}
& D_{B k}^{h}\left(M_{p}, A_{p}\right) \Pi_{B}^{h}+D_{B k}^{l}\left(M_{p}, A_{p}\right) \Pi_{B}^{l} \leq \frac{\alpha(1-\beta)}{\alpha(1-\beta)+(1-\alpha) \beta} \tau G \widehat{q} \widehat{\Pi}_{B} ; \\
& D_{G 1}^{h}\left(M_{p}, A_{p}\right) \Pi_{G}^{h}+D_{G 1}^{l}\left(M_{p}, A_{p}\right) \Pi_{G}^{l} \leq \frac{\alpha(1-\beta)}{\alpha(1-\beta)+(1-\alpha) \beta} \tau G \widehat{q} \widehat{\Pi}_{G},
\end{aligned}
$$

where $\widehat{\Pi}_{T} \equiv \lambda \Pi_{T}^{h}+(1-\lambda) \Pi_{T}^{l}$ is a $T$ provider's expected payoff from serving a consumer in period two.

On the left-hand sides of (5) and (6) are the equilibrium dumping costs in period one of $B k$ and $G 1$ providers, respectively. According to (2), the masses of $h$ and $l$ consumers that a provider has to dump to obtain $\left(M_{p}, A_{p}\right)$ depend on both his quality type $T$ and demand $q$. If $M_{p}=q$, a $T q$ provider dumps no consumer in equilibrium and has no profitable deviation. If $M_{p}<q$, the provider's most profitable deviation is to serve all consumers in period one. However, he will be perceived as a bad type and lose consumers in period two.

On the right-hand sides of (5) and (6) are the penalties of deviations for $B k$ and $G 1$ providers, respectively. In a partial pooling equilibrium, consumers in period two infer that a provider with report $\left(M_{p}, A_{p}\right)$ is a good type with probability $\frac{\alpha(1-\beta)}{\alpha(1-\beta)+(1-\alpha) \beta}$, where $\alpha$ and $1-\beta$ are respectively the prior probabilities that a provider's quality type is $G$ and demand is 1 . The consumers also believe that a provider with any offequilibrium report is a bad type with probability one. Thus, a provider who unilaterally deviates expects to lose $\frac{\alpha(1-\beta)}{\alpha(1-\beta)+(1-\alpha) \beta} \tau G \widehat{q}$ consumers in period two. I let $(k-1)<G \bar{\tau} \widehat{q}$. The inequality ensures the Perfect Report benchmark is not always the unique equilibrium outcome of the game. I also rule out the corner solution where a provider is willing to dump all $l$ consumers in period one by assuming

$$
G \bar{\tau} \widehat{q} \widehat{\Pi}_{T}<(1-\lambda) q \Pi_{T}^{l}
$$

for both $G q$ and $B k$ providers. Finally, the reports $\left(M_{p}, A_{p}\right)$ also have to be infeasible for a $B 1$ provider. The following proposition characterizes partial pooling equilibria with the assumption that condition (3) holds.

Proposition 1 A continuum of partial pooling equilibria exists if $\tau_{p}^{*} \leq \tau<\bar{\tau}$, where $\tau_{p}^{*}$ is given by (8). In the least-dumping-cost equilibrium, a G1 provider serves all his consumers in period one, and a Bk provider dumps $k-1$ of his period-one consumers. The least-cost equilibrium defeats all other partial pooling equilibria.

Figure 2 illustrates the $(M, A)$ pairs that can be supported as the partial pooling equilibrium reports 


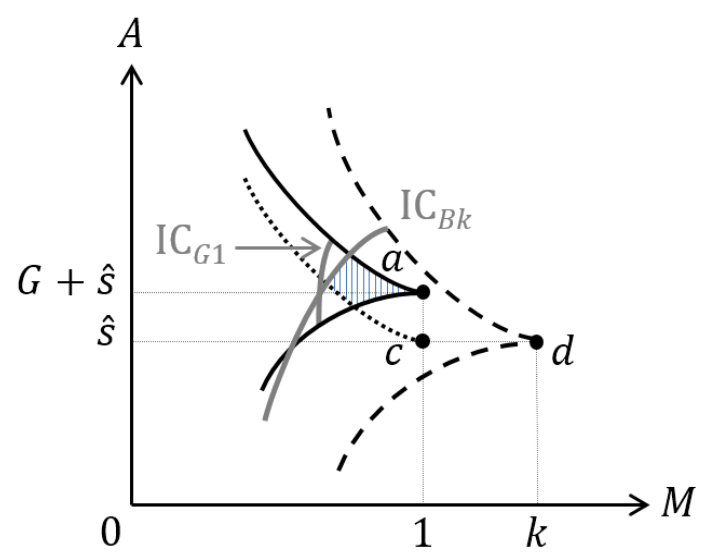

Figure 2: Partial pooling equilibrum reports

of $B k$ and $G 1$ providers. This is the shaded region in the diagram. First, an equilibrium report has to be feasible for a $G 1$ provider to obtain. Therefore, the region is bounded by the two solid curves above and below. When condition (3) in Lemma 1 holds, the reports in the region are also feasible for a $B k$ provider, hence the dashed curve in the diagram is not binding. To make the report infeasible for a $B 1$ provider, the region is also bounded by the dotted curve in the southwest.

Now consider the incentive constraints of $B k$ and $G 1$ providers, conditions (5) and (6), in Figure $2 .{ }^{24}$ Both constraints place restrictions on the maximum masses of consumers that the providers can dump in equilibrium. However, a $B k$ provider has to dump more consumers to obtain any report $\left(M_{p}, A_{p}\right)$ that is feasible for a $G 1$ provider. Therefore, the existence of partial pooling equilibria depends only on the position of (5) relative to point $a$. In the figure, partial pooling equilibria exist because the constraint is on the left-hand side of point $a$. Substituting $M=1$ and $A=G+\widehat{s}$ into (5), I find that partial pooling equilibria exist if $\tau_{p}^{*} \leq \tau<\bar{\tau}$, where

$$
\tau_{p}^{*} \equiv \frac{(k-1) \widehat{\Pi}_{B}+\frac{G}{h-l}\left(\Pi_{B}^{l}-\Pi_{B}^{h}\right)}{\frac{\alpha(1-\beta)}{\alpha(1-\beta)+(1-\alpha) \beta} G \widehat{q} \widehat{\Pi}_{B}} .
$$

Partial pooling equilibria exist only if consumers are responsive to quality difference $\left(\tau_{p}^{*} \leq \tau\right)$. This is because the bigger is $\tau$, the more consumers a deviating provider loses in period two. The value of $\tau_{p}^{*}$ depends on both provider and consumer attributes. The denominator is from the right-hand side of (5). It

\footnotetext{
${ }^{24}$ Figure 2 and Figure 3 are drawn with the assumption that $\omega\left(v^{l}-v^{h}\right)>0$, and hence the incentive constraints are curved.
} 
shows that $\tau_{p}^{*}$ is decreasing in a $B k$ provider's expected cost of deviation. The numerator shows that $\tau_{p}^{*}$ is increasing in $\frac{G}{h-l}\left(\Pi_{B}^{l}-\Pi_{B}^{h}\right)$. This is because the bigger is $\frac{G}{h-l}$, the more $l$ consumers a $B k$ provider has to dump to mimic a $G 1$ provider. The difference, $\Pi_{B}^{l}-\Pi_{B}^{h}$, accounts for the incremental cost of dumping more $l$ consumers from a $B k$ provider's perspective. However, partial pooling equilibria cannot exist $\left(\bar{\tau} \leq \tau_{p}^{*}\right)$ when $\Pi_{B}^{l}-\Pi_{B}^{h}$ is very big, or $\frac{\alpha(1-\beta)}{\alpha(1-\beta)+(1-\alpha) \beta}$ is very small.

In Figure 2 where $\tau_{p}^{*} \leq \tau<\bar{\tau}$, each $(M, A)$ pair in the shaded region corresponds to the partial pooling equilibrium report of an equilibrium. I now apply the undefeated equilibrium refinement to select the most plausible equilibrium report. An equilibrium in this game is defeated if: (i) in the equilibrium there exist an off-equilibrium report $(\widetilde{M}, \widetilde{A})$ that is obtained by a $B k$ provider, a $G 1$ provider, or both in another equilibrium; (ii) the payoffs of all providers who obtain $(\widetilde{M}, \widetilde{A})$ in the alternative equilibrium are weakly higher than their payoffs in the original equilibrium, and strictly higher for at least one of them; and (iii) the consumers' beliefs about a provider with report $(\widetilde{M}, \widetilde{A})$ in the original and alternative equilibria are different. $^{25}$

These criteria permit the equilibrium represented by point $a$ in Figure 2 to defeat all other partial pooling equilibria. This is because point $a$ allows both $B k$ and $G 1$ providers to minimize their respective dumping costs in the shaded region. Therefore, any equilibrium is defeated if the consumers' beliefs after observing the report at point $a$ are more pessimistic than the beliefs on the equilibrium path. To obtain the report at point $a$, a $G 1$ provider always serves all his consumers, and a $B k$ provider dumps $k-1$ of his consumers in period one. By condition (1), the masses of $l$ and $h$ consumers a $B k$ provider dumps in period one are $(k-1)(1-\lambda)+\frac{G}{h-l}$ and $(k-1) \lambda-\frac{G}{h-l}$, respectively. ${ }^{26}$

\footnotetext{
${ }^{25}$ Because I focus on symmetric equilibria, the reports $(\widetilde{M}, \widetilde{A})$ of Providers $x$ and $y$, which are off equilibrium path in the original equilibrium, must also be symmetric. Moreover, the incentives of $B 1$ and $G k$ providers can be ignored because they serve all consumers in every equilibrium.

${ }^{26}$ In the Supplement, I show that the least-cost equilibrium cannot be eliminated by the intuitive criterion if a sufficient condition holds. However, the intuitive criterion can also fail to eliminate other partial pooling equilibria.
} 


\section{Separating equilibria}

In a separating equilibrium, each provider's quality type is fully revealed through his performance report. The period-two outcomes in any informative equilibrium and the Perfect Report benchmark are identical. Because dumping is costly, no provider is willing to manipulate his report by dumping consumers in period one, and to be perceived as a bad type in period two. Thus in any separating equilibrium, a $B k$ provider always serves all consumers in period one. The question remains: Does a $G 1$ provider always serves all his consumers?

Let the equilibrium report of a $G 1$ provider be $\left(M_{G 1}, A_{G 1}\right)$ and that it is not feasible for a $B 1$ provider to obtain the report. I first construct separating equilibria with the consumers' beliefs that a provider with any off-equilibrium report is a bad type. Given this belief, the following inequalities summarize the inter-period incentive trade-offs of $B k$ and $G 1$ providers, respectively:

$$
\begin{aligned}
D_{B k}^{h}\left(M_{G 1}, A_{G 1}\right) \Pi_{B}^{h}+D_{B k}^{l}\left(M_{G 1}, A_{G 1}\right) \Pi_{B}^{l} & \geq \tau G \widehat{q} \widehat{\Pi}_{B} ; \\
D_{G 1}^{h}\left(M_{G 1}, A_{G 1}\right) \Pi_{G}^{h}+D_{G 1}^{l}\left(M_{G 1}, A_{G 1}\right) \Pi_{G}^{l} & \leq \tau G \widehat{q} \widehat{\Pi}_{G} .
\end{aligned}
$$

The best unilateral deviations are not profitable if both conditions (9) and (10) hold.

First consider (9). In any separating equilibrium, a $B k$ provider serves all his consumers in period one. With the pessimistic consumers' beliefs, a $B k$ provider's best unilateral deviation is to obtain report $\left(M_{G 1}, A_{G 1}\right)$. The deviating $B k$ provider could raise his expected demand in period two by $\tau G \widehat{q}$, irrespective of his opponent provider's quality type. ${ }^{27}$ The right-hand side of (9) describes the benefit of this deviation. But the $B k$ provider has to dump some of his consumers in period one in order to obtain $\left(M_{G 1}, A_{G 1}\right)$. The left-hand side of (9) describes the cost.

Now consider (10). In a separating equilibrium, a $G 1$ provider dumps $D_{G 1}^{s}\left(M_{G 1}, A_{G 1}\right)$ of his $s$ consumers in period one. If $M_{G 1}=1$, a $G 1$ provider dumps no consumer and has no profitable deviation. If $M_{G 1}<1$, a $G 1$ provider's best unilateral deviation is to serve all his consumers in period one. The left-hand side of

\footnotetext{
${ }^{27}$ The expected demand increases from $(1-\tau G) \widehat{q}$ to $\widehat{q}$ if the opponent is a $G$ type, and from $\widehat{q}$ to $(1+\tau G) \widehat{q}$ if the opponent is a $B$ type; see Table 2 . The change in expected demand of a $G 1$ provider in the next paragraph is analogous.
} 
(10) describes the benefit of serving more period-one consumers. However, the $G 1$ provider will be perceived as a bad type in period two. The right-hand side of (10) describes the cost of losing consumers in period two. The following proposition characterizes the separating equilibria with the assumption that condition (3) holds.

Proposition 2 A continuum of separating equilibria always exists. At each $\tau$, the least-dumping-cost equilibrium defeats all other separating equilibria. Suppose $\tau \leq \tau_{s}^{*}$ where $\tau_{s}^{*}$ is given by (11). The providers always serve all consumers. Suppose $\tau_{s}^{*}<\tau<\bar{\tau}$. A G1 provider dumps a proportion of his l consumers in period one to satisfy (9) as an equality.

Figure 3 illustrates the construction of separating equilibria. First consider the left panel. The shaded region shows the combinations of $M$ and $A$ that can be supported as a $G 1$ provider's equilibrium report $\left(M_{G 1}, A_{G 1}\right)$. Starting from point $a$, the two solid curves bound the combinations of $M$ and $A$ that are feasible for a $G 1$ provider to obtain. Constraint (10) restricts the maximum masses of consumers that a $G 1$ provider can dump in equilibrium. Constraint (9) restricts the minimum masses of consumers that a $G 1$ provider has to dump to deter a $B k$ provider from mimicking him. In this diagram $\tau \leq \tau_{s}^{*}$, where

$$
\tau_{s}^{*} \equiv \frac{(k-1) \widehat{\Pi}_{B}+\frac{G}{h-l}\left(\Pi_{B}^{l}-\Pi_{B}^{h}\right)}{G \widehat{q} \widehat{\Pi}_{B}} .
$$

Because consumers are less responsive to quality difference, (9) is not binding, and is on the left-hand side of point $a$ in the diagram.

The right panel of Figure 3 illustrates a case where $\tau_{s}^{*}<\tau<\bar{\tau}$. When consumers are responsive to quality difference, (9) binds. In the diagram, any combination of $M$ and $A$ to the left of the $B k$ provider's incentive constraint cannot be supported as a $G 1$ provider's equilibrium report. Consumers' responsiveness also shifts (10) to the right. In the diagram, the shaded region is now bounded by the dotted curve to make $\left(M_{G 1}, A_{G 1}\right)$ infeasible for a $B 1$ provider. Finally, if $\Pi_{B}^{l}-\Pi_{B}^{h}$ in $(9)$ is so big that $\bar{\tau} \leq \tau_{s}^{*}$, a $B k$ provider will not find it profitable to mimic a $G 1$ provider at any degree of demand responsiveness.

Figure 3 also shows the results of undefeated equilibrium refinement when $\tau_{s}^{*}<\bar{\tau}$. Here, as in Spence's 

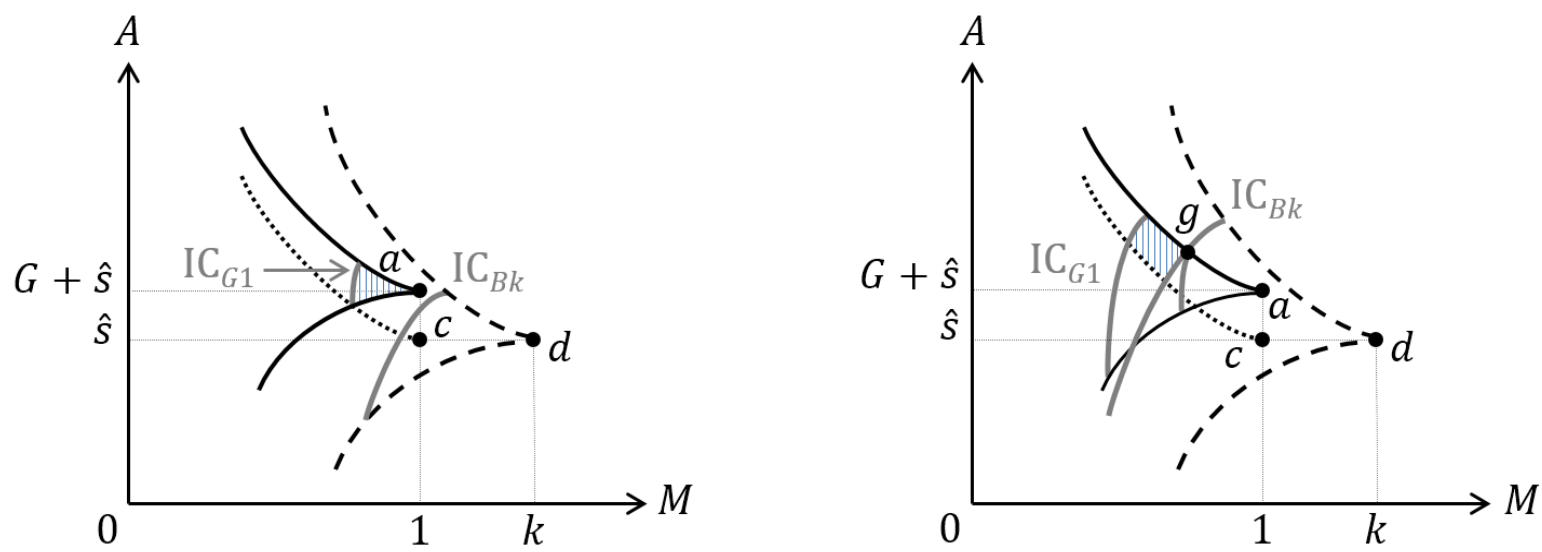

Figure 3: Separating equilibrium reports

(1973) education signalling game, the least-cost equilibrium defeats all other equilibria at every $\tau .^{28}$ In the left panel where $\tau \leq \tau_{s}^{*}$, a $G 1$ provider accepts all consumers in the least-cost equilibrium, and point $a$ represents his equilibrium report. In the right panel where $\tau_{s}^{*}<\tau<\bar{\tau}$, the least-cost equilibrium report $\left(M_{G 1}, A_{G 1}\right)$ must satisfy (9) as an equality. By condition (4), a good provider has a steeper iso-cost curve. Therefore, the report that minimizes a $G 1$ provider's equilibrium dumping cost is at point $g$. In this equilibrium, a $G 1$ provider dumps only $l$ consumers to raise his average outcome and separate from a $B k$ provider. The expression of $D_{G 1}^{l}\left(M_{G 1}, A_{G 1}\right)$ is given by (A1) in the Appendix.

\section{$5 \quad$ Provider strategic response and consumer welfare}

\section{Demand for and supply of quality}

I now relate consumer equilibrium welfare to the demand responsiveness, $\tau$, and the probability that a provider is a good type, $\alpha .^{29}$ By Proposition 1, the least-cost partial pooling equilibrium exists only if $\tau_{p}^{*} \leq \tau<\bar{\tau}$. By Proposition 2, a least-cost separating equilibrium exists at each $\tau$ and dumping occurs only if $\tau_{s}^{*}<\tau<\bar{\tau}$. Because $\tau_{s}^{*}=\frac{\alpha(1-\beta)}{\alpha(1-\beta)+(1-\alpha) \beta} \tau_{p}^{*}$, the Perfect Report benchmark is achieved only if $\tau \leq \tau_{s}^{*}$.

\footnotetext{
${ }^{28}$ The intuitive criterion also eliminates all but the least-cost separating equilibrium at every $\tau$. The proof is standard and omitted.

${ }^{29}$ In the model, a provider's payoffs consist of his consumers' net payoffs. Therefore, I consider consumer welfare rather than total welfare to avoid the problem of double counting. See, for example, Hammond (1987) and Milgrom (1993).
} 


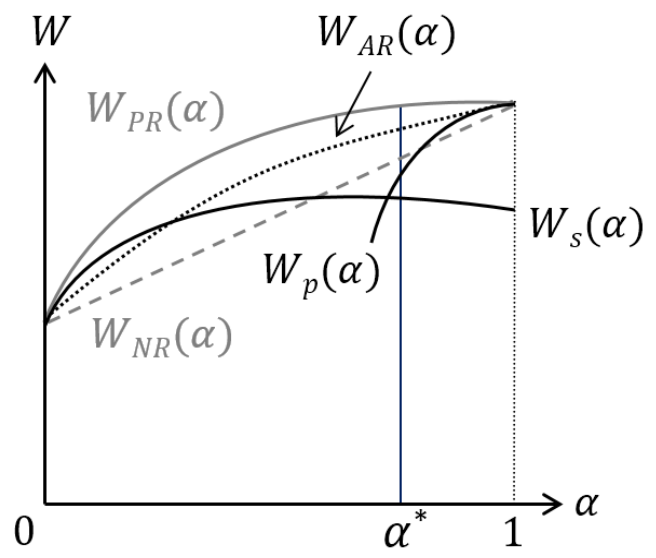

Figure 4: Consumer welfare in the benchmarks and undefeated equilibria

When $\tau_{s}^{*}<\tau<\bar{\tau}$, the benchmark is unattainable. Here, the undefeated equilibrium refinement can be further applied to the two classes of least-cost equilibria to sharpen the welfare analysis.

Proposition 3 The Perfect Report benchmark is achieved only if $\tau \leq \tau_{s}^{*}$. Suppose $\tau_{s}^{*}<\tau<\bar{\tau}$, so that the first best cannot be an equilibrium. At every $\tau$, there is an $\alpha^{*}, 0<\alpha^{*}<1$, such that the least-cost separating equilibrium defeats the least-cost partial pooling equilibrium if $\alpha<\alpha^{*}$, and the least-cost partial pooling equilibrium defeats the least-cost separating equilibrium if $\alpha^{*}<\alpha$.

Figure 4 compares the expected consumer welfare in the least-cost equilibria with the benchmark cases at different $\alpha$ and a fixed $\tau$, where $\tau_{s}^{*}<\tau<\bar{\tau}$. The upper solid curve, $W_{P R}(\alpha)$, represents consumer welfare under Perfect Report. The dashed curve, $W_{N R}(\alpha)$, represents consumer welfare under No Report. Welfare in both benchmarks is increasing in $\alpha$. However, $W_{P R}(\alpha)$ is higher than $W_{N R}(\alpha)$ at every $\alpha$ by $2 \alpha(1-\alpha) \tau G^{2} \widehat{q}$, the welfare gain from quality information dissemination in period two. ${ }^{30}$

The solid curve $W_{p}(\alpha)$ represents consumer welfare in the least-cost partial pooling equilibrium, which exists only if $\alpha$ is big. Comparing $W_{p}(\alpha)$ to the Perfect Report benchmark, there are two sources of welfare loss. First, consumers in period two cannot distinguish $B k$ and $G 1$ providers from their common reports

\footnotetext{
${ }^{30}$ In the expression, $2 \alpha(1-\alpha)$ is the probability that Providers $x$ and $y$ are of different quality types, $\tau G \widehat{q}$ is the expected mass of period-two consumers who switch from a bad type to a good type upon learning the providers' types, and $G$ is the welfare gain of these consumers.
} 
$\left(M_{p}, A_{p}\right) .{ }^{31}$ Second, a $B k$ provider dumps $k-1$ of his consumers in period one to obtain $\left(M_{p}, A_{p}\right)$. The solid curve $W_{s}(\alpha)$ represents consumer welfare in the least-cost separating equilibrium. Period-two welfare in this equilibrium and under Perfect Report are identical. However, a $G 1$ provider dumps $D_{G 1}^{l}\left(M_{G 1}, A_{G 1}\right)$ of his period-one $l$ consumers in equilibrium. Hence, the difference between $W_{s}(\alpha)$ and $W_{P R}(\alpha)$ is increasing in $\alpha .^{32}$

Now consider the undefeated equilibrium refinement when both classes of least-cost equilibria exist. According to (5), a $B k$ provider's gain from pooling with a $G 1$ provider outweighs the cost of dumping $k-1$ of his consumers. Therefore, a $B k$ provider always prefers the partial pooling equilibrium to the separating equilibrium. On the other hand, a $G 1$ provider's preference between the two equilibria is determined by the value of $\alpha$. When $\alpha$ is smaller than the critical value $\alpha^{*}$ in Proposition 3, the incremental benefit from separation $\frac{(1-\alpha) \beta}{\alpha(1-\beta)+(1-\alpha) \beta} \tau G \widehat{q} \widehat{\Pi}_{G}$ (the difference between the right-hand sides of (10) and (6)) is larger than the corresponding dumping cost $D_{G 1}^{l}\left(M_{G 1}, A_{G 1}\right) \Pi_{G}^{l}$ (which is independent of $\alpha$, see (A1) in the Appendix). Thus, a $G 1$ provider strictly prefers the separating report $\left(M_{G 1}, A_{G 1}\right)$ to the partial pooling report $\left(M_{p}, A_{p}\right)$, and the least-cost partial pooling equilibrium is defeated. However, a $G 1$ provider's benefit from separation must be smaller than the corresponding dumping cost when $\alpha$ is larger than $\alpha^{*}$. Here, the partial pooling report $\left(M_{p}, A_{p}\right)$, which is preferred by both $B k$ and $G 1$ providers to their separating reports, enables the least-cost partial pooling equilibrium to defeat the least-cost separating equilibrium.

This critical value $\alpha^{*}$ is characterized in (A4) in the Appendix and illustrated in Figure 4. In the figure, the average quality of providers is low on the left-hand side of $\alpha^{*}$, and $W_{s}(\alpha)$ depicts consumer welfare in the unique undefeated separating equilibrium. On the right-hand side of $\alpha^{*}$, the average quality is high and $W_{p}(\alpha)$ shows consumer welfare in the unique undefeated partial pooling equilibrium. ${ }^{33}$ In general, the value of $\alpha^{*}$ is different from the $\alpha$ at which $W_{p}(\alpha)$ and $W_{s}(\alpha)$ intersect. This is because $\alpha^{*}$ is based on the payoffs

\footnotetext{
${ }^{31}$ The welfare gain from quality information dissemination in a partial pooling equilibrium is $2 \alpha(1-$ $\alpha) \tau G \widehat{q}\left[\beta^{2}\left(G-\frac{\alpha(1-\beta)}{\alpha(1-\beta)+(1-\alpha) \beta} G\right)+\beta(1-\beta) G+(1-\beta)^{2} \frac{\alpha(1-\beta)}{\alpha(1-\beta)+(1-\alpha) \beta} G\right]=2 \alpha(1-\alpha) \tau G^{2} \widehat{q} \frac{\alpha(1-\beta)^{2}+(1-\alpha) \beta^{2}}{\alpha(1-\beta)+(1-\alpha) \beta}$.

${ }^{32}$ Suppose some of the $l$ consumers receive a negative payoff from the service, ceteris paribus. Consumer welfare increases when the providers dump these consumers. Hence, when there are sufficient negative-payoff consumers in the market, the least-cost equilibrium welfare $W_{p}(\alpha)$ and $W_{s}(\alpha)$ can be higher than $W_{P R}(\alpha)$ at some $\alpha$.

${ }^{33}$ The two least-cost equilibria are both undefeated at $\alpha^{*}$.
} 
of a $G 1$ provider, whereas $W_{p}(\alpha)$ and $W_{s}(\alpha)$ are the welfare of consumers served by all providers. Finally, the comparison between consumer welfare in equilibrium and under No Report depends on the values of quality premium $G$, and consumer welfare loss from being dumped $U_{T}^{s}$. Figure 4 considers a case where $G$ is small relative to $U_{B}^{s}$. Hence, $W_{p}(\alpha)$ and $W_{s}(\alpha)$ in the figure are lower than $W_{N R}(\alpha)$ in the intermediate range of $\alpha \cdot{ }^{34}$

\section{Provider motivation}

I next relate the least-cost equilibria and consumer welfare to $\omega$, the weight that a provider assigns to his consumers' net payoffs.

Corollary 1 Suppose $v^{h}<v^{l}$. If $\omega$ decreases, the values of $\tau_{p}^{*}$ and $\tau_{s}^{*}$ decrease, and there is more dumping in the least-cost separating equilibria when $\tau>\tau_{s}^{*}$.

When $\omega$ decreases, a provider puts less weight on his consumers' net payoffs $T+v^{s}$ and more weight on his own profits $P$. This lowers a provider's relative cost of dumping $l$ consumers, who benefit more from the services when $v^{h}<v^{l}$, and rotates the incentive constraints in Figures 2 and 3 counterclockwise. In particular, a lower $\omega$ allows a $B k$ provider to dump more $l$ consumers to pool with, or mimic, a $G 1$ provider at every $\tau$. This lowers the values of $\tau_{p}^{*}$ and $\tau_{s}^{*}$ in Propositions 1 and 2 . When $\tau>\tau_{s}^{*}$, a $G 1$ provider has to dump more $l$ consumers to deter mimicry in the least-cost separating equilibrium. ${ }^{35}$ In terms of consumer welfare, the two benchmark cases are independent of $\omega$. However, a decrease in $\omega$ extends $W_{p}(\alpha)$ in Figure 4 to the left through its effect on $\tau_{p}^{*}$. A lower $\omega$ also downshifts $W_{s}(\alpha)$ in the figure because of the increase in dumping.

Corollary 1 shows that although providers' intrinsic motivation can mitigate dumping, it does not change the least-cost equilibrium outcomes qualitatively. This is because under performance reporting, the incentive

\footnotetext{
${ }^{34}$ The dotted curve $W_{A R}(\alpha)$ in Figure 4 will be defined and analyzed in Section 7.

${ }^{35}$ An increase in $P$ rotates the incentive constraints in the same way as a decrease in $\omega$. However, an increase in $P$ in period one only raises the absolute costs of dumping, and hence, raises the values of $\tau_{p}^{*}$ and $\tau_{s}^{*}$ and lowers the value of $D_{G 1}^{l}\left(M_{G 1}, A_{G 1}\right)$.
} 
trade-offs that determine the equilibrium outcomes are inter-period in nature. ${ }^{36}$ Nevertheless, the effect of providers' motivation on the value of $\alpha^{*}$ in Proposition 3 is generally ambiguous. This is because a lower $\omega$ reduces both the incremental gain from separation and the corresponding dumping cost of a $G 1$ provider.

Now suppose $\omega$ is fixed and $v^{h}$ increases. This raises a provider's expected payoff in period two, and lowers his cost of dumping $l$ consumers relative to $h$ consumers in period one. The two effects rotate the incentive constraints in Figures 2 and 3, and alter the least-cost equilibria in the same way as a decrease in $\omega$ in Corollary 1 as long as $\omega\left(v^{l}-v^{h}\right)>0 .{ }^{37,38}$ Using (4), I can further consider the case where $\omega\left(v^{l}-v^{h}\right)<0$. Here, the providers' incentive constraints will become downward sloping in the figures. This will not affect the level of the least-cost partial pooling equilibrium report. However, in the least-cost separating equilibria where the $B k$ provider's incentive constraint binds, a $G 1$ provider will deter a $B k$ provider's mimicry by dumping $h$ consumers to lower his average service outcome. This appears implausible.

Finally, consider the limiting case where $\omega=0$. When the providers are purely profit maximizing, the incentive constraints in Figures 2 and 3 become vertical. Propositions 1 to 3 continue to hold. However, the expressions for $\tau_{p}^{*}$ and $\tau_{s}^{*}$ in (8) and (11) can be respectively simplified to $\frac{k-1}{G \widehat{q}} / \frac{\alpha(1-\beta)}{\alpha(1-\beta)+(1-\alpha) \beta}$ and $\frac{k-1}{G \widehat{q}}$. The long expression for $D_{G 1}^{l}\left(M_{G 1}, A_{G 1}\right)$ in the Appendix can also be simplified to $\tau G \widehat{q}-(k-1){ }^{39}$ I will focus on this case in the next section to keep the analysis tractable.

\footnotetext{
${ }^{36}$ If the providers are purely altruistic as defined by Andreoni (1989), a $B k$ who assigns a sufficiently big weight to consumer welfare cannot benefit from mimicking or pooling with a $G 1$ provider. In this case, the Perfect Report benchmark is the unique equilibrium at every $\tau$.

${ }^{37}$ This is because $\frac{\partial \tau_{p}^{*}}{\partial \omega}=\frac{\partial \tau_{p}^{*}}{\partial v^{h}}\left[-\frac{\left(v^{l}-v^{h}\right) P}{\omega \Pi_{B}^{l}}\right]$ (and note that $\left.\tau_{s}^{*}=\frac{(1-\alpha) \beta}{\alpha(1-\beta)+(1-\alpha) \beta} \tau_{p}^{*}\right)$, and $\frac{\partial D_{G 1}^{l}}{\partial \omega}=\frac{\partial D_{G 1}^{l}}{\partial v^{h}}\left[-\frac{\left(v^{l}-v^{h}\right) P}{\omega \Pi_{B}^{l}}\right]$.

${ }^{38}$ Alternatively, let the providers' profits from serving $l$ and $h$ consumers be $P^{l}$ and $P^{h}$, respectively. Keeping $P^{h}$ fixed, an increase in $P^{l}$ rotates the incentive constraints in Figures 2 and 3 clockwise. Hence, allowing providers to earn more from serving $l$ consumers can mitigate dumping incentives.

${ }^{39}$ This is a result of the tie-breaking rule I introduced in Section 3 before Lemma 2 . The rule makes $D_{G 1}^{l}\left(M_{G 1}, A_{G 1}\right)$ continuous in $\omega$ when $\omega$ approaches one.
} 


\section{Adjusted average performance report}

\section{Statistical adjustment}

So far, I have illustrated how providers can make use of consumer heterogeneity to game their performance reports in equilibrium. In practice, many reporting agencies address consumer heterogeneity by statistically adjusting performance reports. These statistical techniques are employed to make a provider's average service outcome independent of his consumers' characteristics. The merit of statistical adjustment is well received. If the adjustment can account for the differences in consumer characteristics, a performance report will be more informative about a provider's quality. A provider will have less incentive to dump consumers who can dampen his average performance. This section analyzes providers' dumping behavior under adjusted average outcome reporting. I will show that informative statistical adjustments can lead to less informative reports, more dumping, or both.

Statistical adjustments separate consumer characteristics and provider quality in two steps: First, a reporting agency uses a statistical model to estimate a consumer's service outcome with a set of observable variables. The statistical model predicts the consumer's expected service outcome that is independent of her provider's identity. Second, a provider's adjusted average outcome is calculated as the difference between the actual and expected average outcomes of his consumers. For example, reporting agencies in the health care sector use age, gender, and comorbidities to estimate patients' mortality rates; they then report the difference between the expected and actual mortality rates as a provider's risk-adjusted performance. Agencies in the education sector use past exam scores to estimate students' current test scores; they then report the difference between the expected and actual test scores as a teacher's value-added score.

In the following, I model an observable variable (such as age or past test score) as an exogenous public signal about a consumer's characteristic (such as health status or learning ability). ${ }^{40}$ The signal has two possible realizations $\mu \in\{L, H\}$. An $l$ consumer and an $h$ consumer obtain signal realization $L$ with probabilities $1-\varepsilon$ and $\varepsilon$, respectively. I assume $0 \leq \varepsilon<\frac{1}{2}$. By the Bayes' rule, a consumer with signal $L$ is

\footnotetext{
${ }^{40}$ This is in line with the theoretical literature on statistical adjustment (see, for example, Glazer and McGuire, $2000)$.
} 
more likely to have characteristic $l$ than a consumer with signal $H$. Hence, the signal is informative about consumer characteristic that is not observable by the reporting agency. The parameter $\varepsilon$ can be interpreted as the signal's noise. The values of $L$ and $H$ are the expected service outcomes of the consumers with those signal realizations. I let $l \leq L<H \leq h$.

A provider's adjusted performance report is about the mass of consumers he has served in period one, $M$, and the adjusted average outcome of these consumers, $A A$. The adjusted average outcome is the average difference between the actual outcome $(T+s)$ and expected outcome $\mu$ of the served consumers. The values of $\varepsilon$ and $\mu$ are commonly known. In each period, each provider observes both the characteristic and signal of any consumer who requests his service. A provider then decides whether to dump each of his consumers as before. However, a provider can now base his dumping decision on a consumer's signal realization too. If a $T q$ provider dumps $D_{T q}^{s \mu}$ of his $s$ consumers with signal $\mu$ in period one, his performance report is

$(M, A A)=\left(\begin{array}{c}\left\{\lambda(1-\varepsilon) q-D_{T q}^{h H}\right\}(T+h-H)+\left\{\lambda \varepsilon q-D_{T q}^{h L}\right\}(T+h-L) \\ q-D_{T q}^{h H}-D_{T q}^{h L} \\ -D_{T q}^{l H}-D_{T q}^{l L}\end{array}, \frac{+\left\{(1-\lambda) \varepsilon q-D_{T q}^{l H}\right\}(T+l-H)+\left\{(1-\lambda)(1-\varepsilon) q-D_{T q}^{l L}\right\}(T+l-L)}{q-D_{T q}^{h H}-D_{T q}^{h L}-D_{T q}^{l H}-D_{T q}^{l L}}\right)$.

In period two, consumers infer a provider's quality type from his performance report $(M, A A)$. Other aspects of the game are as in Section 2. The Perfect Report and No Report benchmarks in Section 3 continue to apply.

\section{Perfect adjustment}

I begin with a benchmark case where the signal is perfectly informative about the consumer characteristic.

Lemma 3 Suppose $\varepsilon=0, L=l$, and $H=h$. The Adjusted Average Outcome Reporting game has a unique equilibrium in which a provider's adjusted average outcome is his quality type $T$, and the Perfect Report benchmark is always achieved.

If $\varepsilon=0$, all $l$ consumers receive signal $L$, and all $h$ consumers receive signal $H$. Suppose $L=l$ and $H=h$. When a provider serves a consumer, the adjusted outcome is always $T$. In other words, the perfect statistical adjustment completely separates provider quality from consumer characteristic. There is no room 
for report manipulation. In equilibrium, the providers always serve all consumers, as under the Perfect Report benchmark.

\section{Imperfect adjustment and gaming feasibility}

If $0<\varepsilon<\frac{1}{2}$, the signal is still informative about the consumer characteristic. However, $h$ and $l$ consumers now receive signal realizations $L$ and $H$, respectively, with probability $\varepsilon$. If a $T q$ provider serves all his consumers in period one, his performance report is now $M=q$ and $A A=T+\widehat{s}-\widehat{\mu}$, where $\widehat{\mu} \equiv \lambda\{(1-$ $\varepsilon) H+\varepsilon L\}+(1-\lambda)\{(1-\varepsilon) L+\varepsilon H\}$ is the expected value of the signal. Because of the differences in quality $T$ and demand $q$, it is still impossible for $B 1$ and $G k$ providers to pool with other providers in equilibrium.

Now consider $B k$ and $G 1$ providers. Under average outcome reporting, Lemma 1 introduces condition (3), which allows a $B k$ provider to mimic a $G 1$ provider who serves all consumers. The equilibrium analysis in Section 4 shows that condition (3) is necessary for the Perfect Report benchmark to be unattainable. Now return to adjusted average outcome reporting. The following lemma considers the condition under which a $B k$ provider can mimic a $G 1$ provider who serves all consumers.

Lemma 4 Continue to assume that $k-1<k(1-\lambda)$. Under adjusted outcome reporting, the report of a $G 1$ provider who serves all his consumers can be mimicked by a Bk provider who dumps some of his consumers if

$$
G \leq(k-1) \lambda(h-l)+[\operatorname{Min}\{(k-1)(\lambda \varepsilon+(1-\lambda)(1-\varepsilon)),(1-\lambda) \varepsilon-(k-1) \lambda(1-\varepsilon)\} \times(H-L)] .
$$

The value of the square-bracket term in (13) is negative if and only if $\varepsilon<\varepsilon^{*} \equiv \frac{(k-1)}{(k-1)+(1-\lambda) / \lambda}$.

A provider's adjusted average outcome depends on the signal realizations of the consumers whom he chooses to serve. This accounts for the extra square-bracket term in (13) compared to (3) in Lemma 1. Because of the quality difference, a $B k$ provider has to mimic a $G 1$ provider who serves all consumers by raising his adjusted average from $\widehat{s}-\widehat{\mu}$ to $G+\widehat{s}-\widehat{\mu}$. Consider his consumers' adjusted outcomes, $s-\mu$. They are ranked as $l-H<l-L<h-H<h-L$ because $l \leq L<H \leq h$. Therefore, a $B k$ provider can raise his adjusted average the most by first dumping his $l$ consumers with signal $H$, and then dumping his $l$ 
consumers with signal $L .^{41}$

To compare the effects of dumping an additional $l$ consumer on a $B k$ provider's average and adjusted average, I differentiate $A$ in (1) with respect to $D_{B k}^{l}$ and $A A$ in (12) with respect to $D_{B k}^{l H}$ and $D_{B k}^{l L}$ for a fixed $\varepsilon$. I then evaluate the derivatives at the same mass of dumped consumers under the two regimes and get

$$
\frac{\partial A}{\partial D_{B k}^{l}}<\left.\frac{\partial A A}{\partial D_{B k}^{l H}}\right|_{D_{B k}^{l}=D_{B k}^{l H}} \text { and } \frac{\partial A}{\partial D_{B k}^{l}}>\left.\frac{\partial A A}{\partial D_{B k}^{l L}}\right|_{D_{B k}^{l}=k(1-\lambda) \varepsilon+D_{B k}^{l L}} .
$$

The first inequality in (14) indicates that signal $H$ makes dumping an $l$ consumer a more effective way of gaming at the margin. This is because the reporting agency expects a consumer with signal $H$ to have an above-average service outcome $(\widehat{\mu}<H)$, and hence, dumping the consumer raises a $B k$ provider's adjusted average more. The second inequality of (14) indicates that it is less effective to game the adjusted average at the margin by dumping an $l$ consumer with signal $L$. This is because the reporting agency expects a consumer with signal $L$ to have a below-average service outcome $(L<\widehat{\mu}) .{ }^{42}$

The size of $\varepsilon$ determines the total effect of dumping a given proportion of $l$ consumers on a $B k$ provider's adjusted average, as compared to his unadjusted average. Figure 5 visualizes this comparison. In the left panel, the left and right axes denote $A$ and $A A$, respectively. The curve $\bar{A}_{B k}$ represents the average outcome that a $B k$ provider can obtain by dumping $l$ consumers. The curve $\overline{A A}_{B k}$ represents the adjusted average outcome that the same provider can obtain by dumping $l$ consumers. The shapes of the two curves illustrate the inequalities in condition (14). $\overline{A A}_{B k}$ is steeper than $\bar{A}_{B k}$ only if a $B k$ provider dumps less than $k(1-\lambda) \varepsilon$ of his $l$ consumers.

The left panel is a case where $\varepsilon<\varepsilon^{*}$. Here, the public signal is sufficiently informative about the consumer characteristic. The proportion of $l$ consumers who receive signal $H$ is small relative to the difference in demand $k-1$. The value of the square-bracket term in (13) is negative, hence (13) implies (3) in Lemma 1. In the diagram, point $a$ is below $\bar{A}_{B k}$ but above $\overline{A A}_{B k}$. A $B k$ provider can mimic a $G 1$ provider's average

\footnotetext{
${ }^{41}$ Moreover, as long as (7) holds, a $B k$ provider would have no incentive to dump $h$ consumers to raise his adjusted average.

${ }^{42}$ The two inequalities in (14) are $\frac{k \lambda(h-l)}{M^{2}}<\frac{k[\lambda(h-l)+\{\lambda \varepsilon+(1-\lambda)(1-\varepsilon)\}(H-L)]}{M^{2}}$ and $\frac{k \lambda(h-l)}{M^{2}}>\frac{\lambda k[(h-l)-(1-\varepsilon)(H-L)]}{M^{2}}$, respectively.
} 

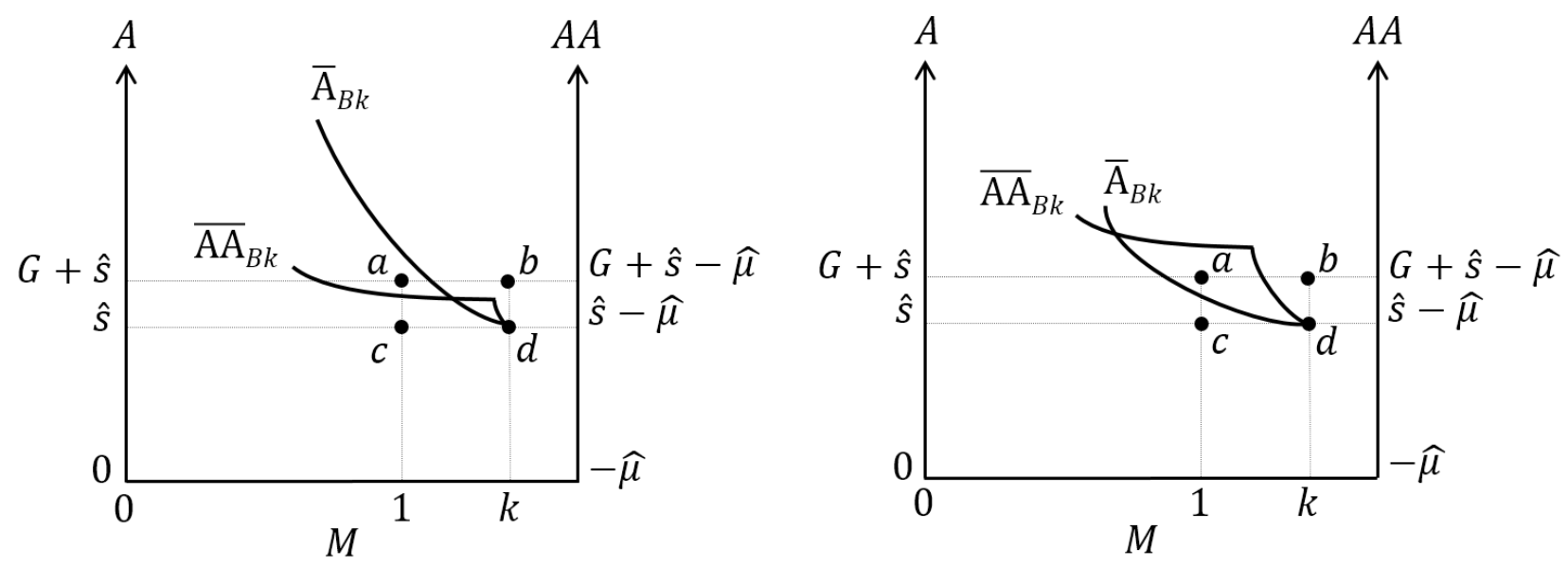

Figure 5: Feasibility of gaming in the two reporting regimes

outcome but not his adjusted average outcome at that point.

The right panel is a case where $\varepsilon^{*}<\varepsilon$. This case is nontrivial because $k-1<k(1-\lambda)$ implies $\varepsilon^{*}<\frac{1}{2}$. Here, the public signal is still informative about the consumer characteristic. However, the signal noise is sufficiently big, so that the value of the square-bracket term in (13) is positive, and (3) in Lemma 1 implies (13). In the diagram, point $a$ is below $\overline{A A}_{B k}$ but above $\bar{A}_{B k}$. A $B k$ provider can mimic a $G 1$ provider's adjusted average outcome but not his average outcome at point $a$.

The value of $\varepsilon^{*}$ in Lemma 4 is independent of the expected service outcomes $H$ and $L$. However, their difference, $H-L$, determines the absolute value of the square-bracket term in (13). The larger is $H-L$, the larger are the absolute differences between $\bar{A}_{B k}$ and $\overline{A A}_{B k}$ in the two panels of Figure 5 , hence the more likely that a $B k$ provider is able to mimic a $G 1$ provider in only one of the two reporting regimes. I will next compare the equilibria under the two reporting regimes.

\section{Imperfect adjustment and equilibria}

The constructions of equilibria under adjusted outcome reporting are essentially the same as before. In a partial pooling equilibrium, the report $\left(M_{p}, A A_{p}\right)$ has to be feasible for both $B k$ and $G 1$ providers. In a separating equilibrium, the report $\left(M_{G 1}, A A_{G 1}\right)$ has to be feasible for a $G 1$ provider. Finally, in all the incentive constraints in Section 4 , the expressions $D_{T q}^{s}(M, A)$ have to be replaced by $\left\{D_{T q}^{s L}(M, A A)+\right.$ 
$\left.D_{T q}^{s H}(M, A A)\right\}$ that are derived from (12). Section 5 has shown that the least-cost equilibria under average outcome reporting have the same qualitative properties as long as $\omega\left(v^{l}-v^{h}\right)$ is nonnegative. To economize notation and facilitate comparison, I will characterize equilibria under adjusted outcome reporting by setting $\omega=0$. I continue to apply the undefeated equilibrium refinement to multiple equilibria.

Proposition 4 Suppose condition (13) holds and $\omega=0$. If

$$
H-L<\frac{1}{1-\varepsilon}\left[(h-l)-\frac{G(1-(1-\lambda) \varepsilon)}{(k-1) \lambda}\right]
$$

then the statements of Propositions 1, 2, and 3 continue to characterize the undefeated partial pooling and separating equilibria under adjusted outcome reporting.

The proposition says that when a $B k$ provider can mimic a $G 1$ provider who accepts all consumers, the two reporting regimes lead to the same levels of dumping and report informativeness as long as inequality (15) holds. Figure 6 explains the intuition. In the left panel, the dashed curve $\overline{A A}_{B k}$ has the same interpretation as in Figure 5. The solid curve $\overline{A A}_{G 1}$ represents the adjusted average that a $G 1$ provider can obtain by dumping $l$ consumers, and it has the same shape as $\overline{A A}_{B k}$.

Given $\omega=0$, the two vertical solid lines, $\mathrm{IC}_{\mathrm{Bk}}$ Pool and $\mathrm{IC}_{\mathrm{Bk}}$ Sep respectively represent (5) and (9) under average outcome reporting. Point $a$ is the partial pooling equilibrium average outcome report $\left(M_{p}, A_{p}\right)$ as in Figure 2, and point $g$ is the separating equilibrium average outcome report $\left(M_{G 1}, A_{G 1}\right)$ as in Figure 3 (however, the incentive constraint is vertical here but curved in Figures 2 and 3). Because the cost and expected benefit of dumping are the same under the two reporting regimes, the two vertical solid lines also represent a $B k$ provider's incentive constraints under adjusted outcome reporting.

The left panel is a case where inequality (15) holds. This sufficient condition guarantees that $\overline{A A}_{B k}$ is higher than $\overline{A A}_{G 1}$ in the relevant range. Here, under adjusted outcome reporting, point $a$ also represents the least-cost partial pooling report $\left(M_{p}, A A_{p}\right)$ that defeats all other equilibrium reports in its class. In addition, point $h$ in the panel represents the least-cost separating report $\left(M_{G 1}, A A_{G 1}\right)$ that satisfies the tie-breaking rule. Because points $g$ and $h$ are on the same vertical line, a $G 1$ provider dumps the same proportion of 

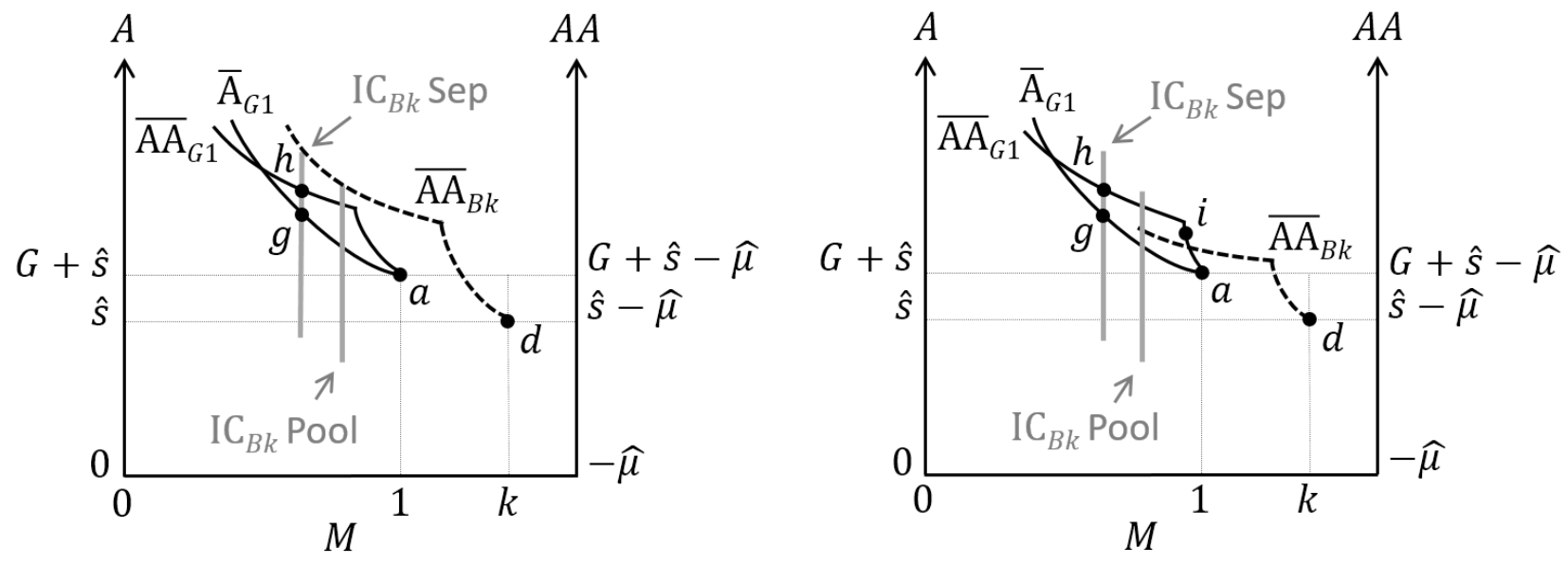

Figure 6: Comparing equilibrium reports and dumpings in the two reporting regimes

consumers in equilibrium under the two regimes. The consumer welfare analysis in Section 5 follows. ${ }^{43}$

Condition (15) requires the range of expected service outcomes, $H-L$, to be smaller than a critical value. This critical value is $(h-l)-\frac{G}{(k-1) \lambda}$ when $\varepsilon$ is zero (which is strictly positive if (3) holds), and is increasing in $\varepsilon$. Hence, the bigger is $\varepsilon$, the more likely that (15) holds. The right panel of Figure 6 shows an example where (15) is violated. In the panel, $\overline{A A}_{B k}$ and $\overline{A A}_{G 1}$ intersect on the left-hand side of $\mathrm{IC}_{\mathrm{Bk}}$ Sep. Point $h$ can no longer be a separating equilibrium report. A $G 1$ provider can achieve separation by the report at point $i$, which is infeasible for a $B k$ provider. Because a $G 1$ provider dumps less consumers at point $i$ than at point $g$, the report at point $i$ may defeat the partial pooling equilibrium report at point $a$ even if $\alpha$ is larger than $\alpha^{*}$ in Proposition 3.

Corollary 2 Consider the undefeated equilibria under the two reporting regimes when $\omega=0$. (i) If (3) is satisfied but (13) is not, dumping and pooling can occur only if the reports are unadjusted. (ii) If both (3) and (13) are satisfied and (15) holds, the two regimes lead to the same levels of dumping, report informativeness, and consumer welfare. (iii) If (13) is satisfied but (3) is not, dumping and pooling can occur only if the reports are adjusted.

\footnotetext{
${ }^{43}$ When $\omega\left(v^{l}-v^{h}\right)>0$, the slopes of a provider's iso-dumping-cost curves are generally different under the two reporting regimes. This leads to a small difference in the $M_{G 1}$ and the least-cost separating equilibrium welfare under the two regimes.
} 
This corollary follows directly from Lemma 4 and Proposition 4. According to Lemma 4, (3) holds and (13) fails simultaneously only if $\varepsilon$ is small. This is the only case where statistical adjustment can unambiguously eliminate gaming. When $\varepsilon$ is big and (13) is satisfied, the power of statistical adjustment is counter-balanced by the providers' strategy response. Statistical adjustment can completely fail to mitigate dumping, as illustrated by the left panel of Figure 6. It can even lead to more dumping in equilibrium by allowing a $B k$ provider to mimic a $G 1$ provider, as illustrated in the right panel of Figure 5.

\section{Discussion and concluding remarks}

\section{Testable predictions}

I now consider some testable predictions of the model. I first show that the least-cost partial pooling and separating equilibria under both reporting regimes generate different correlations between service volumes and average outcomes.

Corollary 3 Compared to the Perfect Report and No Report benchmarks, the least-cost partial pooling equilibria under both reporting regimes generate more positive correlations between service volumes and average outcomes in the market; whereas the least-cost separating equilibria with dumping under both reporting regimes generate more negative correlations between service volumes and average outcomes in the market.

These correlations occur because in a least-cost partial pooling equilibrium, it is a $B k$ provider who reduces volume to raise his average outcome, but in a least-cost separating equilibrium, it is a $G 1$ provider who reduces volume to raise his average outcome. The surgery volume-outcome relationship is well studied in health services research. For example, Marcin et al. (2008) find that after California replaced its voluntary cardiac surgery outcome report cards with mandatory report cards in 2003, the correlation between volume and survival rate in the state has became more positive. This observation is consistent with a least-cost partial pooling equilibrium outcome. ${ }^{44}$

\footnotetext{
${ }^{44}$ There is a large literature that uses correlation between risk occurrence and coverage to test for the presence of adverse selection in insurance markets. For recent reviews, see, for example, Cohen and Siegelman (2010), and Chiappori and Salanie (2013).
} 
Consider next the observable implications when providers game their adjusted average performance reports. According to condition (14), a provider can raise his adjusted average outcome more by dumping $l$ consumers with signal $H$ than by dumping those with signal $L$. The following corollary shows that if consumers with signal $H$ are indeed more likely to be dumped, the market expected and actual average outcomes will change in the opposite directions.

Corollary 4 Let $D_{l \mu}$ be the mass of $l$ consumers with signal $\mu \in\{L, H\}$ who are being dumped in the market. Compared to the Perfect Report and No Report benchmarks, the market actual and expected average outcomes raises and falls, respectively, if

$$
\frac{D_{l L}}{\lambda \varepsilon+(1-\lambda)(1-\varepsilon)}<\frac{D_{l H}}{\lambda(1-\varepsilon)+(1-\lambda) \varepsilon} .
$$

Recall that the adjusted average outcome is the difference between the actual and expected average outcomes, and that the expected outcome is the average $\mu$ of the served consumers. When providers dump $l$ consumers, the actual average outcome increases irrespective of the consumers' signal realizations. If inequality (16) also holds, consumers with signal $H$ are more likely to be dumped. ${ }^{45}$ Therefore, the average $\mu$ of the served consumers will decrease with dumping, and the divergence between actual and expected outcomes will lead to a significant increase in the adjusted average outcome. This phenomenon has been observed in New York State after the introduction of cardiac surgery report cards in 1989 (Hannan et al., 1994). The mechanism in the corollary raises caution about interpreting an increase in adjusted average as a clear evidence of quality improvement. ${ }^{46}$

\section{Reporting average outcomes only}

Returning to Table 1. The analysis so far has focused on report categories 1 and 2. Now consider report categories 3 and 4 . Specifically, let the reporting agency report only the average outcome $A$, or adjusted outcome $A A$, but not the mass $M$. In either case, consumers in period two have to infer a provider's quality

\footnotetext{
${ }^{45}$ The denominators on the left-hand and right-hand sides of (16) are the proportions of consumers who obtain signal $L$ and $H$, respectively.

${ }^{46}$ An alternative explanation is that hospitals have upcoded patient severities. However, an audit by the New York State Department of Health in 1992 showed no significant difference between the expected survival rates based on the hospitals' coding and the audited coding (Hannan et al., 1994).
} 
type from a single number. This is also essentially identical to an agency rewarding or punishing providers based on their average outcomes or adjusted average outcomes. ${ }^{47}$

When the agency does not report $M, G k$ and $G 1$ providers who serve all consumers obtain the same level of performance report $(A=G+\widehat{s}$ or $A A=G+\widehat{s}-\widehat{\mu})$. Characterizing the equilibria is beyond the scope of this section. The following corollary focuses on whether $B k$ and $B 1$ providers can mimic $G k$ and G1 providers or not.

Corollary 5 Continue to assume $k-1<k(1-\lambda)$. Suppose the reporting agency reports average outcome $A$ only. The reports of $G k$ and $G 1$ providers who serve all consumers are $G+\widehat{s}$. This level of report can be mimicked by either Bk or B1 provider who dumps some of his consumers if

$$
G \leq(1-\lambda)(h-l)
$$

This inequality implies (3) in Lemma 1. Now suppose the agency switches to report adjusted average outcome $A A$ only. The reports of $G k$ and $G 1$ providers who serve all consumers become $G+\widehat{s}-\widehat{\mu}$. Either Bk or B1 provider can obtain this level of report by dumping less of his consumers if $\varepsilon>\varepsilon_{A A} \equiv \frac{G}{G+(1-\lambda)(h-l)}$.

Consider a $B k$ provider first. The intuition of Corollary 5 is illustrated in the right panel of Figure 5 . There, $\bar{A}_{B k}$ is lower than $G+\widehat{s}$ at $M=1$. When the agency reports $(M, A)$, it is not feasible for a $B k$ provider to mimic a $G 1$ provider's report at point $a$. However, the $\bar{A}_{B k}$ curve reaches $A=G+\widehat{s}$ at an $M$ below 1 . When the agency reports only $A$, mimicry becomes feasible. Now let the agency switch to reporting only $A A$. The value of $\varepsilon_{A A}$ in the corollary is below $\frac{1}{2}$ when inequality (17) holds. Recall that the right panel of Figure 5 is a case where $\varepsilon$ is big, so the signal is sufficiently noisy. The $\overline{A A}_{B k}$ curve in the diagram shows that a $B k$ provider can reach $A A=G+\widehat{s}-\widehat{\mu}$ at an $M$ above 1 . Thus the imperfect statistical adjustment allows a $B k$ provider to mimic a $G 1$ provider by dumping less consumers.

Now consider a $B 1$ provider, who can never mimic another $T q$ provider when the agency reports either $(M, A)$ or $(M, A A)$. When $M$ is not reported, a $B 1$ provider can obtain any report that is feasible for a $B k$

\footnotetext{
${ }^{47}$ In addition to the JTPA program in Table 1, the Centers for Medicare and Medicaid Services also introduced minimum adjusted average survival standards on organ transplantations in 2007. Stith and Hirth (forthcoming) find that kidney transplant centers have responded to the standards by dumping sicker patients and performing fewer transplantations.
} 
provider by dumping his consumers proportionately. Therefore, the conditions in Corollary 5 apply to a $B 1$ provider as well.

\section{Alternative reporting scheme}

Although gaming becomes easier when the agency reports only $A$ or $A A$, the agency can potentially eliminate dumping by reporting less information than $(M, A)$ and $(M, A A)$. Consider the following alternative reporting scheme. The agency reports that a provider is Above Average if $M \geq k$ and $A \geq G+\widehat{s}$, Below Average if $M \leq 1$ and $A \leq \operatorname{Min}\left\{h, \frac{\widehat{s}-(1-M) l}{M}\right\}$, and Average otherwise. The following corollary characterizes the equilibrium outcome and consumer welfare under the scheme.

Corollary 6 A unique undefeated equilibrium exists under the alternative reporting scheme. In equilibrium, the providers always serve all consumers, the reporting agency reports that a Gk provider is Above Average, a B1 provider is Below Average, and both $G 1$ and Bk providers are Average. Consumer equilibrium welfare $W_{A R}(\alpha)$ is higher than $W_{N O}(\alpha)$ and $W_{p}(\alpha)$ defined in Section 5 at all $\alpha$. Moreover, $W_{A R}(\alpha)$ is higher than $W_{s}(\alpha)$ when $\alpha$ is big.

The expression $\operatorname{Min}\left\{h, \frac{\widehat{s}-(1-M) l}{M}\right\}$ is the highest possible $A$ that a $B 1$ provider can obtain by dumping $l$ consumers at every $M \leq 1$. Therefore, the scheme always designates a $B 1$ provider as Below Average, irrespective of his service decisions. On the other hand, $M \geq k$ and $A \geq G+\widehat{s}$ are only feasible for a $G k$ provider who serves all his consumers. This threshold also disables both $G 1$ and $B k$ providers from improving consumer perceptions by dumping consumers in period one. Because no provider can benefit from dumping, the scheme designates both $G 1$ and $B k$ providers as Average, and conveys the same quality information as the partial pooling reports do in Section 4.

The curve $W_{A R}(\alpha)$ in Figure 4 illustrates consumer welfare under the alternative report scheme. In the figure, $W_{A R}(\alpha)$ is always lower than $W_{P R}(\alpha)$ and higher than $W_{N R}(\alpha)$. This is because the three cases involve no dumping in period one, so they are ranked only by consumer welfare in period two. In the same figure, $W_{A R}(\alpha)$ and $W_{p}(\alpha)$ share the same level of consumer welfare in period two. However, dumping in period one makes $W_{p}(\alpha)$ always lower than $W_{A R}(\alpha)$ when partial pooling equilibria exist. Finally, the 
comparison of $W_{A R}(\alpha)$ and $W_{s}(\alpha)$ involves an inter-period trade-off. Consumers in period two learn more from the separating equilibrium reports than from the alternative report scheme, but a $G 1$ provider has to dump consumers in period one in the most efficient separating equilibrium when $\tau_{s}^{*}<\tau<\bar{\tau}$. Figure 4 shows that this trade-off depends on the value of $\alpha$. When $\alpha$ is big the welfare loss from dumping is big and the value of quality information is small (see Section 5), hence $W_{A R}(\alpha)$ is higher than $W_{s}(\alpha)$.

Although the alternative reporting scheme may appear to be artificial, it can be interpreted as a mechanism that aggregates multidimensional information into coarse ratings. ${ }^{48}$ Corollary 6 , along with Corollaries 2 and 5, show that the design of optimal reporting strategy is an important area for future research.

\section{Matching}

In the model, each provider receives service requests from consumers with an identical distribution of characteristic. Therefore, the providers' performance reports (with and without statistical adjustment) are perfect indicators of their quality types if they serve all consumers. In the area of education, Lang (2010) discusses how teacher performance reports are biased when good and poor teachers are matched with students with different distributions of learning ability. Biased performance reports can make gaming more feasible. To see this, let the proportions of $h$ consumers who visit $G 1$ and $B k$ providers in period one be $\widetilde{\lambda}$ and $\lambda$, respectively. Under average outcome reporting, a $B k$ provider can mimic a $G 1$ provider who serves all consumers if $G \leq(k-1) \lambda(h-l)+(\lambda-\widetilde{\lambda})(h-l)$. This inequality is implied by (3) in Lemma 1 as long as $\widetilde{\lambda} \leq \lambda$, so that a $G 1$ provider is matched with less $h$ consumers and more $l$ consumers.

Policy makers and academic researchers often suggest that statistical adjustment can create a level playing field when providers are matched with different consumer distributions. This argument may not hold when providers are strategic. Consider adjusted average outcome reporting and let $k-1<k(1-\lambda) \varepsilon$ for simplicity. ${ }^{49}$

\footnotetext{
${ }^{48}$ Dubey and Geanakoplos (2010) show that coarse exam grading is optimal when students value status. Harbaugh and Rasmusen (2014) find that coarse quality certification can be more informative because it attracts more providers to apply. In both articles information is unidimensional.

${ }^{49}$ The inequality says that the mass of $l$ consumers with signal $H$ who visit a provider $B k$ in period one is larger than $k-1$.
} 
Using the same notation $\widetilde{\lambda}$. A $B k$ provider can mimic a $G 1$ provider who serves all consumers if

$$
G \leq(k-1) \lambda(h-l)+(k-1)[\lambda \varepsilon+(1-\lambda)(1-\varepsilon)](H-L)+(\lambda-\tilde{\lambda})[(h-l)-(1-2 \varepsilon)(H-L)] .
$$

Because $\varepsilon<1 / 2$ and $H-L<h-l$, the above inequality is also implied by (13) in Lemma 4 as long as $\tilde{\lambda} \leq \lambda$.

\section{Concluding remarks}

This article has developed a model to study the feasibility and incentive of provider gaming under different performance reporting regimes. Sections 2 to 6 have analyzed report categories 1 and 2 in Table 1 . I showed that report category 1 can achieve the Perfect Report benchmark only when the intensity of competition is low. When competition is strong, these reports may not eliminate quality information asymmetry, but always lead to dumping. I then compared the equilibrium outcomes under report categories 1 and 2 . I found that inaccurate statistical adjustments can result in less informative reports but more dumping.

This section has further considered the testable implications of provider gaming, and how the feasibility of gaming can be affected by the content of performance reports and provider-consumer matching. These results can be applied to the study and development of performance reports for physicians, hospitals, teachers, schools, and other public service providers.

Throughout the article, I have assumed that a provider's quality type is his private information. This formulation ignores the possibility that a provider can learn his performance from his report and subsequently improve his service quality, which is the key concern in Kolstad (2013). The design of performance reports when providers can behave constructively and strategically remains an important task for future research. 


\section{Appendix}

This appendix contains omitted proofs.

Proof of Lemma 1: If a $G 1$ provider serves all his consumers, his report is $(1, G+\widehat{s})$. Let $k-1<k(1-\lambda)$ and substitute $D_{B k}^{h}=0, D_{B k}^{l}=k-1$ into (1). A $B k$ provider's report is now $(1, k \lambda(h-l)+l)$. The average $k \lambda(h-l)+l$ is not lower than $G+\widehat{s} \equiv G+\lambda h+(1-\lambda) l$ if $G \leq(k-1) \lambda(h-l)$.

Proof of Proposition 1: (a) Existence. A partial pooling equilibrium report must be feasible for both $G 1$ and $B k$ providers, and satisfy conditions (5) and (6). Moreover, a $B 1$ provider must find it either unprofitable or infeasible to obtain the report.

I first show that the report $(1, G+\widehat{s})$ is feasible for both $G 1$ and $B k$ providers, and infeasible for a $B 1$ provider. By condition (2), a $G 1$ provider can obtain report $(1, G+\widehat{s})$ by serving all his consumers, and a $B k$ provider can obtain the same report by choosing

$$
D_{B k}^{h}(1, G+\widehat{s})=(k-1) \lambda-\frac{G}{h-l} \text { and } D_{B k}^{l}(1, G+\widehat{s})=(k-1)(1-\lambda)+\frac{G}{h-l} .
$$

Upon simplification, $0<D_{B k}^{h}(1, G+\widehat{s})$ and $D_{B k}^{l}(1, G+\widehat{s})<k(1-\lambda)$ if $k-1<k(1-\lambda)$ and $G \leq(k-1) \lambda(h-l)$. Therefore, the report $(1, G+\widehat{s})$ is feasible for a $B k$ provider if Lemma 1 holds. Finally, the report $(1, G+\widehat{s})$ is never feasible for a $B 1$ provider because his average outcome is $\widehat{s}$ when $M=1$.

I now show that partial pooling equilibria exist only if $(1, G+\widehat{s})$ can be supported as an equilibrium report. First, the value of the left-hand side of $(6)$ is zero at and only at $(1, G+\widehat{s})$. Second, according to (4), the slope of a $B k$ provider's iso-dumping-cost curve is $\frac{d A}{d M}=\frac{\Pi_{B}^{l}(h-A)+\Pi_{B}^{h}(A-l)}{M\left(\Pi_{B}^{l}-\Pi_{B}^{h}\right)}$. And according to (1), the slope of the lower bound of a $G 1$ provider feasibility $(M, A)$ set (the upper-sloping solid curve starting from point $a$ in Figure 2) is $-\frac{d A}{d D_{G 1}^{h}}=\frac{d A}{d M}=\frac{(1-\lambda)(h-l)}{M^{2}}$. Upon simplification, the iso-dumping-cost curve is steeper because $0 \leq \omega\left(v^{l}-v^{h}\right)$ and Lemma 1 imply $1>\frac{\Pi_{B}^{l}-\Pi_{B}^{h}}{\Pi_{B}^{l}} \frac{G}{h-l}$. Hence, the value of the left-hand side of $(5)$ at $(1, G+\widehat{s})$ must also be the lowest among all reports that are feasible for a $G 1$ provider.

Because $(6)$ is always nonbinding at $(1, G+\widehat{s})$, the existence of partial pooling equilibria depends only 
on (5). Substitute $D_{B k}^{h}(1, G+\widehat{s})$ and $D_{B k}^{l}(1, G+\widehat{s})$ into (5). The constraint is satisfied if $\tau_{p}^{*} \leq \tau<\bar{\tau}$, where $\tau_{p}^{*}$ in (8) is the value of $\tau$ that holds (5) as an equality.

(b) Refinement. I have shown that $(1, G+\widehat{s})$ minimizes the left-hand sides of both (5) and (6) among all possible partial pooling reports. Therefore, in any undefeated partial pooling equilibrium, the consumers' beliefs upon observing $(1, G+\widehat{s})$ must be $\frac{\alpha(1-\beta)}{\alpha(1-\beta)+(1-\alpha) \beta}$. The beliefs eliminate any equilibrium in which the partial pooling report is not $(1, G+\widehat{s})$.

Proof of Proposition 2: (a) Existence. In a separating equilibrium, a $G 1$ provider's report $\left(M_{G 1}, A_{G 1}\right)$ must satisfy (10) and be feasible for a $G 1$ provider to obtain. The report must also satisfy (9) or be infeasible for a $B k$ provider. Finally, the same report must be infeasible or unprofitable for a $B 1$ provider to obtain.

Incentive constraints (9) and (10) restrict, respectively, the minimum and maximum proportions of consumers that a $G 1$ provider can dump. The strictly-positive slopes of these curves in the report space $(M, A)$ are given by (4). Because $0 \leq \omega\left(v^{l}-v^{h}\right)$, (9) is never steeper than (10). Therefore, separating equilibria exist only if the report that is feasible for a $G 1$ provider, and satisfies (9) with the biggest mass and highest average, can be supported as $\left(M_{G 1}, A_{G 1}\right)$. Because of the quality premium $G$, the report $\left(M_{G 1}, A_{G 1}\right)$ is always infeasible for a $B 1$ provider to obtain. I now characterize this report at every $\tau, \tau<\bar{\tau}$. There are two cases.

Case 1. Condition (9) is satisfied at $(1, G+\widehat{s})$. Because $(1, G+\widehat{s})$ is feasible for a $G 1$ provider and (9) is not binding here, the report can be supported as $\left(M_{G 1}, A_{G 1}\right)$. Substitute $D_{B k}^{h}(1, G+\widehat{s})$ and $D_{B k}^{l}(1, G+\widehat{s})$ into (9). The constraint is satisfied if $\tau \leq \tau_{s}^{*}$, where $\tau_{s}^{*}$ in (11) is the value of $\tau$ that holds (9) as an equality.

Case 2. Condition (9) is not satisfied at $(1, G+\widehat{s})$ or $\tau_{s}^{*}<\tau$. Here a $G 1$ provider must dump consumers to satisfy (9) as an equality. Inequality (7) guarantees that a $B k$ provider would never dump all his $l$ consumers and hence $M_{G 1}>k \lambda$ and $A_{G 1}<h$. Therefore, a $G 1$ provider must dump less than $(1-\lambda)$ of his $l$ consumers to obtain the report $\left(M_{G 1}, A_{G 1}\right)$, which satisfies (9) with the biggest mass and highest average. By condition (1), $D_{G 1}^{l}\left(M_{G 1}, A_{G 1}\right)$ has to satisfy

$$
1-D_{G 1}^{l}=k-D_{B k}^{h}-D_{B k}^{l} \text { and } G+\frac{\lambda h+(1-\lambda) l-D_{G 1}^{l} l}{1-D_{G 1}^{l}}=\frac{k(\lambda h+(1-\lambda) l)-D_{B k}^{h} h-D_{B k}^{l} l}{k-D_{B k}^{h}-D_{B k}^{l}} .
$$


The binding incentive constraint (9) requires $D_{B k}^{h} \Pi_{B}^{h}+D_{B k}^{l} \Pi_{B}^{l}=\tau G \widehat{q} \widehat{\Pi}_{B}$. The three equalities solve for

$$
D_{G 1}^{l}=\left[\tau G \widehat{q} \frac{\widehat{\Pi}_{B}}{\Pi_{B}^{l}}-(k-1)-\frac{\Pi_{B}^{l}-\Pi_{B}^{h}}{\Pi_{B}^{l}}\left((k-1) \lambda-\frac{G}{h-l}\right)\right] /\left[1-\frac{\Pi_{B}^{l}-\Pi_{B}^{h}}{\Pi_{B}^{l}} \frac{G}{h-l}\right]
$$

Finally, constraint (10) is not binding at $D_{G 1}^{l}$ given by (A1) because $\tau G \widehat{q} \widehat{\Pi}_{G} \bar{\Pi}_{G}^{l}-D_{G 1}^{l}=$

$$
\tau G \widehat{q}\left[\frac{\widehat{\Pi}_{G}}{\bar{\Pi}_{G}^{l}}-\frac{\widehat{\Pi}_{B}}{\Pi_{B}^{l}}\right]+\frac{\Pi_{B}^{l}-\Pi_{B}^{h}}{\Pi_{B}^{l}}\left[(k-1) \lambda-\frac{G}{h-l}\right]+\frac{1}{\lambda}\left[(k-1) \lambda-\left(\left[\tau G \widehat{q} \frac{\widehat{\Pi}_{G}}{\Pi_{G}^{l}}\right]\left[\frac{\Pi_{B}^{l}-\Pi_{B}^{h}}{\Pi_{B}^{l}}\right] \lambda\right) \frac{G}{h-l}\right]>0,
$$

where $\Pi_{T}^{s} \equiv \omega\left(T+v^{s}\right)+(1-\omega) P$ and $\widehat{\Pi}_{T} \equiv \lambda \Pi_{T}^{h}+(1-\lambda) \Pi_{T}^{l}$. In (A2), the first squared-bracket term is nonnegative because $0<G$ and $0 \leq \omega\left(v^{l}-v^{h}\right)$. The second squared-bracket term is nonnegative if (3) holds. The expression $\frac{\Pi_{B}^{l}-\Pi_{B}^{h}}{\Pi_{B}^{l}}$ in front of the second and in the third squared-bracket terms is nonnegative and smaller than one because $0 \leq \omega\left(v^{l}-v^{h}\right)$. Moreover, $\tau G \widehat{q} \widehat{\frac{\Pi_{G}}{\Pi_{G}^{l}}}<(1-\lambda)$ according to (7). Therefore, the value of the third squared-bracket term is bigger than $(k-1) \lambda-\frac{G}{h-l}$, which is nonnegative according to Lemma 1.

(b) Refinement. A $B k$ provider incurs no dumping cost in all separating equilibria, and hence, the equilibrium in which a $G 1$ provider obtains the highest payoff must defeat other separating equilibria. When $(1, G+\widehat{s})$ satisfies $(9)$, it is obviously the report that gives the highest payoff to a $G 1$ provider. When $(1, G+\widehat{s})$ fails to satisfy $(9),\left(M_{G 1}, A_{G 1}\right)$ in part (a) is selected to satisfy the constraint with the biggest $M$. By construction, When $0<\omega\left(v^{l}-v^{h}\right)$, a $G 1$ provider's iso-dumping-cost curves are steeper than (9) everywhere. Therefore, $\left(M_{G 1}, A_{G 1}\right)$ in part (a) gives a $G 1$ provider the highest equilibrium payoff at every $\tau$. When $0=\omega\left(v^{l}-v^{h}\right)$, a $G 1$ provider is indifferent among all reports that satisfy (9) as an equality. However, the tie-breaking rule in Section 2 selects $\left(M_{G 1}, A_{G 1}\right)$ in part (a) as the equilibrium report at every $\tau$.

Proof of Proposition 3: Let $\tau_{s}^{*}<\tau<\bar{\tau}$ and that both classes of equilibria exist. By Propositions 1 and 2, the difference between a $G 1$ provider's payoffs in the least-cost separating equilibrium and partial pooling equilibrium is

$$
-D_{G 1}^{l}\left(M_{G 1}, A_{G 1}\right) \Pi_{G}^{l}+\frac{(1-\alpha) \beta}{\alpha(1-\beta)+(1-\alpha) \beta} \tau G \widehat{q} \widehat{\Pi}_{G}
$$

where $D_{G 1}^{l}\left(M_{G 1}, A_{G 1}\right)$ is given by (A1). Because $D_{G 1}^{l}$ is independent of $\alpha$ and $\tau G \widehat{q} \widehat{\frac{\Pi}{G}}_{G}-D_{G 1}^{l}>0$, (A3) is negative if and only if $\alpha^{*}<\alpha$, where

$$
0<\alpha^{*} \equiv \beta\left[\tau G \widehat{q} \widehat{\Pi}_{G}^{l}{ }_{G}^{l}-D_{G 1}^{l}\right] /\left(\beta\left[\tau G \widehat{q} \widehat{\Pi}_{G}^{l}{ }_{G}^{l}-D_{G 1}^{l}\right]+(1-\beta) D_{G 1}^{l}\right)<1
$$


Suppose $0<\alpha<\alpha^{*}$ so that (A3) is positive. If the least-cost partial pooling equilibrium is not defeated by the least-cost separating equilibrium, consumers must believe in the partial pooling equilibrium that a provider with report $\left(M_{G 1}, A_{G 1}\right)$ is a $G 1$ provider with probability one. Therefore, a $G 1$ provider can raise his payoff by moving from the partial pooling report $\left(M_{p}, A_{p}\right)$ to $\left(M_{G 1}, A_{G 1}\right)$. This profitable deviation is a contradiction.

Now suppose $\alpha^{*}<\alpha<1$ so that (A3) is negative. If the least-cost separating equilibrium is not defeated by the least-cost partial pooling equilibrium, consumers must believe in the separating equilibrium that a provider with report $\left(M_{p}, A_{p}\right)$ is a $G 1$ provider with probability $\frac{\alpha(1-\beta)}{\alpha(1-\beta)+(1-\alpha) \beta}$. Thus according to (5) and (A3), both $B k$ and $G 1$ providers' payoffs increase by moving from their respective separating reports to $\left(M_{p}, A_{p}\right)$. Again, a contradiction.

Proof of Corollary 1: First, differentiate $\tau_{p}^{*}$ in (8) with respect to $\omega$ and collect terms, I have

$$
\frac{\partial \tau_{p}^{*}}{\partial \omega}=\left[\left(v^{l}-v^{h}\right) P\right] /\left[\frac{\alpha(1-\beta)}{\alpha(1-\beta)+(1-\alpha) \beta} G \widehat{q} \widehat{\Pi}_{B}\right]<0
$$

Because $\tau_{s}^{*}=\frac{\alpha(1-\beta)}{\alpha(1-\beta)+(1-\alpha) \beta} \tau_{p}^{*}$, the derivative implies $\frac{\partial \tau_{s}^{*}}{\partial \omega}<0$. Second, let $\tau_{s}^{*}<\tau$ and differentiate $D_{G 1}^{l}$ in (A1) with respect to $\omega$. Upon simplification, I have

$$
\frac{\partial D_{G 1}^{l}}{\partial \omega}=-\left(v^{l}-v^{h}\right) P\left[\left((k-1) \lambda-\frac{G}{h-l}\right)+(k-1) \frac{G}{h-l}+\tau G \widehat{q}\left(\lambda-\frac{G}{h-l}\right)\right] /\left[\Pi_{B}^{l}-\left(\Pi_{B}^{l}-\Pi_{B}^{h}\right) \frac{G}{h-l}\right]^{2} .
$$

This derivative is always negative, and it is sufficient to check the first squared-bracket term. According to (3), $(k-1) \lambda-\frac{G}{h-l}$ is nonnegative. Suppose $\frac{G}{h-l} \leq \lambda$. The squared-bracket term is positive because $0<(k-1) \frac{G}{h-l}$. Suppose $\lambda<\frac{G}{h-l}$. By inequality $(7)$ and $(1-\lambda) \Pi_{B}^{l}<\widehat{\Pi}_{B}$, I have $\tau G \widehat{q}<k$. Hence, $(k-1) \frac{G}{h-l}+\tau G \widehat{q}\left(\lambda-\frac{G}{h-l}\right)>k \lambda-\frac{G}{h-l}>(k-1) \lambda-\frac{G}{h-l}$ and the squared-bracket term is positive.

Proof of Lemma 4: Because $l-H<l-L$, a $B k$ provider can raise his adjusted average more by dumping $l$ consumers with signal $H$. Suppose $k-1 \leq k(1-\lambda) \varepsilon$. A $B k$ provider dumps only $l$ consumers with signal $H$ to reach $M=1$. By (12), the provider's adjusted average is $k(\widehat{s}-\widehat{\mu})-(k-1)(l-H)$, where $\widehat{s} \equiv \lambda h+(1-\lambda) l$ and $\widehat{\mu} \equiv \lambda\{(1-\varepsilon) H+\varepsilon L\}+(1-\lambda)\{(1-\varepsilon) L+\varepsilon H\}$. This is not lower than $G+\widehat{s}-\widehat{\mu}$ if

$$
G \leq(k-1)[\lambda(h-l)+\{\lambda \varepsilon+(1-\lambda)(1-\varepsilon)\}(H-L\}]
$$

Next, suppose $k(1-\lambda) \varepsilon<k-1$. Now a $B k$ provider has to dump all his $l$ consumers with signal $H$ and 
$(k-1)-k(1-\lambda) \varepsilon$ of his $l$ consumers with signal $L$ to reach $M=1$. By (13), his adjusted average is $k(\widehat{s}-\widehat{\mu})-k(1-\lambda) \varepsilon(l-H)-\{(k-1)-k(1-\lambda) \varepsilon\}(l-H)$. This is not lower than $G+\widehat{s}-\widehat{\mu}$ if

$$
G \leq(k-1)[\lambda(h-l)+\{(1-\lambda) \varepsilon+(k-1) \lambda(1-\varepsilon)\}(H-L\}] .
$$

The value of the right-hand side of (A6) is smaller than that of (A5) if and only if $k(1-\lambda) \varepsilon<k-1$. Combining the two conditions leads to (13) in the lemma. In (13), $\lambda \varepsilon+(1-\lambda)(1-\varepsilon)$ is always strictly positive, and $(1-\lambda) \varepsilon+(k-1) \lambda(1-\varepsilon)$ is negative if and only if $k(1-\lambda) \varepsilon<k-1$.

Proof of Proposition 4: I first show that if (13) holds and no provider is willing to dump all of his $l$ consumers, condition (15) implies there is no report $(M, A A)$ that is only feasible for a $G 1$ provider. Because of the quality premium $G>0$, any report $(M, A A)$ that is feasible for a $G 1$ provider and has $A A<G+\widehat{s}-\widehat{\mu}$ must also be feasible for a $B k$ provider. Now consider the reports with $A A>G+\widehat{s}-\widehat{\mu}$. Specifically, consider the highest $A A$ that both $G 1$ and $B k$ providers can obtain at different $M$. Because (7) has ruled out the corner solution where a provider dumps all his $l$ consumers, it is sufficient to consider $k \lambda<M \leq 1$. Using (12) to calculate $\overline{A A}_{B k}-\overline{A A}_{G 1}$ at $M, k \lambda<M \leq 1$, I have

$$
\begin{array}{ll}
\frac{1}{M}(k-1)[\lambda(h-l)+\{\lambda \varepsilon+(1-\lambda)(1-\varepsilon)\}(H-L)]-G & \text { if } k(1-(1-\lambda) \varepsilon)<M \leq 1 ; \\
\frac{1}{M}[(k-1) \lambda\{(h-l)-(1-\varepsilon)(H-L)\}-\{1-(1-\lambda) \varepsilon-M\}(H-L)]-G & \text { if } \operatorname{Max}\{1-(1-\lambda) \varepsilon, k \lambda\} \\
\frac{1}{M}[(k-1) \lambda\{(h-l)-(1-\varepsilon)(H-L)\}-\{1-(1-\lambda) \varepsilon-M\}(H-L)]-G & \text { if } k \lambda<M \leq M \leq \operatorname{Min}\{k(1-(1-\lambda) \varepsilon), 1\}
\end{array}
$$

In the first case both $G 1$ and $B k$ providers dump only $l$ consumers with signal $H$. In the second case a $B k$ provider dumps $l$ consumers with both signals, whereas a $G 1$ provider dumps only $l$ consumers with signal $H$. Finally, in the third case both $G 1$ and $B k$ providers dump $l$ consumers with both signals.

Note that $\overline{A A}_{B k}-\overline{A A}_{G 1}$ is positive in case one if (13) in Lemma 4 holds. Moreover, in both case one and case three, $\overline{A A}_{B k}-\overline{A A}_{G 1}$ is always decreasing in $M$ because $H-L \leq h-l$. Therefore, $\overline{A A}_{B k}-$ $\overline{A A}_{G 1}$ can possibly be nonpositive only in case two. Condition (15) says that $\overline{A A}_{B k}-\overline{A A}_{G 1}$ is positive at $M=1-(1-\lambda) \varepsilon$. This condition is sufficient to guarantee that $\overline{A A}_{B k}-\overline{A A}_{G 1}$ is positive in all cases, and that no report $(M, A A)$ is only feasible for a $G 1$ provider.

Now suppose inequalities (13), (15) hold and $\omega=0$. Consider partial pooling equilibria. Following the proof of Proposition 1, it is easy to see that equilibria exist only if $(1, G+\widehat{s}-\widehat{\mu})$ can be supporting as 
$\left(M_{p}, A A_{p}\right)$. When $\omega=0, \Pi_{T}^{h}=\Pi_{T}^{l}=\widehat{\Pi}_{T}$ and $(1, G+\widehat{s}-\widehat{\mu})$ satisfies the modified incentive constraint (5) if $k-1 \leq \frac{\alpha(1-\beta)}{\alpha(1-\beta)+(1-\alpha) \beta} \tau G \widehat{q}$. The last inequality leads to $\tau_{p}^{*}$ that is identical to the one in (8) with $\omega=0$. For refinement, note that when $\Pi_{T}^{h}=\Pi_{T}^{l}=\widehat{\Pi}_{T}$, a provider's dumping cost depends only on the total mass of consumers he dumps. Because $(1, G+\widehat{s}-\widehat{\mu})$ allows both $G 1$ and $B k$ providers to dump the least consumers, the equilibrium with that report defeats all other partial pooling equilibria.

Next, consider separating equilibria. Again, follow the proof of Proposition 2 and consider the equilibrium reports at which a $G 1$ provider dumps the least consumers. When $\omega=0$, the report $(1, G+\widehat{s}-\widehat{\mu})$ satisfies the modified incentive constraint (5) if $k-1 \leq \tau G \widehat{q}$. Therefore, $(1, G+\widehat{s}-\widehat{\mu})$ can be supported as $\left(M_{G 1}, A A_{G 1}\right)$ if $\tau \leq \tau_{s}^{*} \equiv \frac{k-1}{G \widehat{q}}$, and this $\tau_{s}^{*}$ is also the same as the one in (11) with $\omega=0$. If $\tau_{s}^{*}<\tau \leq \bar{\tau}$, the modified incentive constraint (9) must be satisfied as an equality. Here, the tie-breaking rule always selects the report with the highest $A A$. Hence,

$$
D_{G 1}^{l H}\left(M_{G 1}, A A_{G 1}\right)+D_{G 1}^{l L}\left(M_{G 1}, A A_{G 1}\right)=\tau G \widehat{q}-(k-1) .
$$

The modified incentive constraint (10) is not binding at this $\left(M_{G 1}, A A_{G 1}\right)$ because the constraint allows a $G 1$ provider to dump, at most, $\tau G \widehat{q}$ of his consumers. Moreover, the values of $D_{G 1}^{l}$ in (A1) and $D_{G 1}^{l H}+D_{G 1}^{l L}$ in (A7) are identical when $\omega=0$. Finally, at every $\tau$, the equilibrium report characterized above allows a G1 provider to dump the lowest possible proportion of his consumers. Hence, this is the only equilibrium report that satisfies the tie-breaking rule and is not defeated by any other separating equilibrium.

Because $G 1$ and $B k$ providers are dumping the same proportions of consumers in each class of least-cost equilibria under the two reporting regimes. Proposition 3 remains valid under adjusted average outcome reporting.

Proof of Corollary 4: First, the actual average outcomes with and without dumping are

$$
\alpha G+\frac{\lambda h+\left\{(1-\lambda)-D_{l H}-D_{l L}\right\} l}{1-D_{l H}-D_{l L}}>\alpha G+\lambda h+(1-\lambda) l,
$$

where $\alpha G$ is the market average quality premium. The inequality holds as long as $0<D_{l H}+D_{l L}$.

Now consider the expected average outcomes. The expected outcome with dumping is lower than the 
one without dumping if

$$
\frac{\lambda\{\varepsilon L+(1-\varepsilon) H\}+(1-\lambda)\{\varepsilon H+(1-\varepsilon) L\}-D_{l H} H-D_{l L} L}{1-D_{l H}-D_{l L}}>\lambda\{\varepsilon L+(1-\varepsilon) H\}+(1-\lambda)\{\varepsilon H+(1-\varepsilon) L\},
$$

which yields (16) upon simplification.

Proof of Corollary 5: When the agency only reports $A$, a $B q$ provider, $q=1, k$, can obtain report $A=G+\widehat{s}$ by setting $G+\widehat{s}=\frac{q \widehat{s}-D_{B q}^{l}(G+\widehat{s}) l}{q-D_{B q}^{l}(G+\widehat{s})}$ or $D_{B q}^{l}(G+\widehat{s})=\frac{G q}{G+\lambda(h-l)}$. The report $A=G+\widehat{s}$ is feasible for a $B k$ provider if $\frac{G q}{G+\lambda(h-l)} \leq q(1-\lambda)$. This inequality can be simplified to (17) in the corollary. Moreover, (3) implies (17) because $k-1<k(1-\lambda)$ implies $k \lambda<1$, or $(k-1) \lambda<(1-\lambda)$.

Now suppose the agency only reports $A A$. There are two cases to consider. Case 1. A $B q$ provider can obtain report $A A=G+\widehat{s}-\widehat{\mu}$ by dumping only $l$ consumers with signal $H$. In this case $D_{B q}^{l}(G+\widehat{s}-\widehat{\mu})$ is given by $G+\widehat{s}-\widehat{\mu}=\frac{q(\widehat{s}-\widehat{\mu})-D_{B q}^{l}(G+\widehat{s}-\widehat{\mu})(l-H)}{q-D_{B q}^{l}(G+\widehat{s}-\widehat{\mu})}$ or $D_{B q}^{l}(G+\widehat{s}-\widehat{\mu})=\frac{G q}{G+\lambda(h-l)+(H-\widehat{\mu})}$. The last expression is always smaller than $D_{B q}^{l}(G+\widehat{s})=\frac{G q}{G+\lambda(h-l)}$ because $\widehat{\mu}<H$.

Case 2. A $B q$ provider can obtain report $A A=G+\widehat{s}-\widehat{\mu}$ only if he dumps $l$ consumers with both signals. In this case $D_{B q}^{l}(G+\widehat{s}-\widehat{\mu})$ is given by

$$
\begin{aligned}
G+\widehat{s}-\widehat{\mu} & =\frac{1}{k-D_{B q}^{l}(G+\widehat{s}-\widehat{\mu})}\left[q(\widehat{s}-\widehat{\mu})-\left\{D_{B q}^{l}(G+\widehat{s}-\widehat{\mu})-q(1-\lambda) \varepsilon\right\}(l-L)-k(1-\lambda) \varepsilon(l-H)\right] \text { or } \\
D_{B q}^{l}(G+\widehat{s}-\widehat{\mu}) & =q[G-(1-\lambda) \varepsilon(H-L)] /[G+\lambda(h-l)-\{\lambda \varepsilon+(1-\lambda)(1-\varepsilon)\}(H-L)] .
\end{aligned}
$$

Upon simplification, $D_{B q}^{l}(G+\widehat{s}-\widehat{\mu})<D_{B q}^{l}(G+\widehat{s})=\frac{G q}{G+\lambda(h-l)}$ if $\frac{G}{G+(1-\lambda)(h-l)}<\varepsilon$.

Proof of Corollary 6: First, according to (1), only a $G k$ provider can obtain $M \geq k, A \geq G+\widehat{s}$ and be designated as Above Average. Second, again according to (1), a $B 1$ provider will be designated as Below Average, irrespective of his dumping decisions. Finally, according to the same condition, both $G 1$ and $B k$ providers can obtain $M \leq 1$ and $A \leq \operatorname{Min}\left\{h, \frac{\widehat{s}-(1-M) l}{M}\right\}$ and be designated as Below Average by dumping some consumers, but each of them can be designated as Average by serving all consumers.

I now show that in the unique undefeated equilibrium, each $T q$ provider serves all his consumers in period one. First, serving all consumers in period one is the optimal strategy of each $T q$ provider if consumers in period two believe that an Above Average provider is a $G k$ provider with probability one, an Average provider is a $G 1$ provider with probability $\frac{\alpha(1-\beta)}{\alpha(1-\beta)+(1-\alpha) \beta}$ and a $B k$ provider with probability $\frac{(1-\alpha) \beta}{\alpha(1-\beta)+(1-\alpha) \beta}$, and that 
a Below Average provider is a $B 1$ provider with probability one. Therefore, this is indeed an equilibrium. Second, given the construction of the alternative reporting scheme, the equilibrium simultaneously allows each $T q$ provider to have the highest possible designation and demand in period two, and to dump no consumer in period one. Therefore, the equilibrium must defeat all other equilibria.

Consider welfare next. I first use the expressions for welfare in Footnotes 30 and 31 to get

$$
\begin{aligned}
W_{P R}(\alpha)-W_{N R}(\alpha) & =2 \alpha(1-\alpha) \tau G^{2} \widehat{q} \\
W_{P R}(\alpha)-W_{p}(\alpha) & =2 \alpha(1-\alpha) \tau G^{2} \widehat{q} \frac{\beta(1-\beta)}{\alpha(1-\beta)+(1-\alpha) \beta}+2(1-\alpha) \beta\left[D_{B k}^{h} U_{B}^{h}+D_{B k}^{l} U_{B}^{l}\right] \\
W_{P R}(\alpha)-W_{s}(\alpha) & =2 \alpha(1-\beta) D_{G 1}^{l} U_{G}^{l} \\
W_{P R}(\alpha)-W_{A R}(\alpha) & =2 \alpha(1-\alpha) \tau G^{2} \widehat{q} \frac{\beta(1-\beta)}{\alpha(1-\beta)+(1-\alpha) \beta} .
\end{aligned}
$$

From these expressions, it is easy to see that $W_{N R}(\alpha)<W_{A R}(\alpha)<W_{P R}(\alpha)$ at every $\alpha$, and that $W_{p}(\alpha)<$ $W_{A R}(\alpha)$ whenever partial pooling equilibria exist. Now compare $W_{A R}(\alpha)$ with $W_{s}(\alpha)$, the former is higher if

$$
\tau G^{2} \widehat{q} \frac{(1-\alpha) \beta}{\alpha(1-\beta)+(1-\alpha) \beta}-D_{G 1}^{l} U_{G}^{l}<0
$$

This inequality must hold when $\alpha$ is sufficiently close to one. 


\section{References}

Albano, G.L. and Lizzeri, A. "Strategic Certification and Provision of Quality." International Economic Review, Vol. 42 (2001), pp. 267-283.

Andreoni, J. "Giving with Impure Altruism: Applications to Charity and Ricardian Equivalence." Journal of Political Economy, Vol. 97 (1989), pp. 1447-1458.

Bagwell, K. "Signalling and Entry Deterrence: A Multidimensional Analysis." RAND Journal of Economics, Vol. 38 (2007), pp. 670-697.

Bar-Issac, H., Caruana, G., and Cunat, V. "Information Gathering Externalities for a Multi-Attribute Good." Journal of Industrial Economics, Vol. 60 (2012), pp. 162-185.

Brown, J., Duggan, M., Kuziemko, I., and Woolston, W. "How Does Risk Selection Respond to Risk Adjustment? New Evidence from the Medicare Advantage Program." American Economic Review, Vol. 104 (2014), pp. 3335-3364.

Chen, Y. "Multidimensional Signalling and Diversification." RAND Journal of Economics, Vol. 28 (1997), pp. $168-187$.

Chen, Y. "Why are Health Care Report Cards So Bad (Good)?" Journal of Health Economics, Vol. 30 (2011), pp. 575-590.

Chiappori, P.A. and Salanie, B. "Asymmetric Information in Insurance Markets: Predictions and Tests." In G. Dionne, eds., Handbook of Insurance, New York: Springer, 2013.

Cho, I.-K. and Kreps, D.M. "Signaling Games and Stable Equilibria." Quarterly Journal of Economics, Vol. 102 (1987), pp. 179-221.

Cohen, A. and Siegelman, P. "Testing for Adverse Selection in Insurance Markets." Journal of Risk and Insurance, Vol. 77 (2010), pp. 39-84. 
Courty, P., Kim, D.H., and Marschke, G. "Curbing Cream-Skimming: Evidence on Enrolment Incentives." Labour Economics, Vol. 18 (2011), pp. 643-655.

Daughety, A. and Reinganum, J.F. "Competition and Confidentiality: Signaling Quality in a Duopoly when there is Universal Private Information." Games and Economic Behavior, Vol. 58 (2007), pp. 94-120.

Dranove, D., Kessler, D., McClellan, M., and Satterthwaite, M. "Is More Information Better? The Effects of 'Report Cards' on Health Care Providers." Journal of Political Economy, Vol. 111 (2003), pp. $555-588$.

— . and Jin, G.Z. "Quality Disclosure and Certification: Theory and Practice." Journal of Economic Literature, Vol. 48 (2010), pp. 935-963.

Dubey, P. and Geanakoplos, J. "Grading Exams: 100, 99, 98,.. or A, B, C?" Games and Economic Behavior, Vol. 69 (2010), pp. 72-94.

Ederer, F., Holden, R., and Meyer, M. "Gaming and Strategic Opacity in Incentive Provision." Mimeo, Cowles Foundation for Research in Economics, Yale University, 2014.

Ellis, R.P. "Creaming, Skimping and Dumping: Provider Competition on the Intensive and Extensive Margins." Journal of Health Economics, Vol. 17 (1998), pp. 537-555.

Ely, J.C. and Valimaki, J. "Bad Reputation." Quarterly Journal of Economics, Vol. 118 (2003), pp. 785814.

Fong, K. "Evaluating Skilled Experts: Optimal Scoring Rules for Surgeons." Mimeo, Department of Economics, Stanford University, 2009.

Francois, P. "'Public Service Motivation' as an Argument for Government Provision." Journal of Public Economics, Vol. 78 (2000), pp. 275-299.

Glazer, J. and McGuire, T.G. "Optimal Risk Adjustment in Markets with Adverse Selection: An Application to Managed Care." American Economic Review, Vol. 90 (2000), pp. 1055-1071. 
Grossman, S.J. "The Informational Role of Warranties and Private Disclosure about Product Quality." Journal of Law and Economics. Vol. 24 (1981), pp. 461-483.

Hammond, P.J. "Altruism." In J. Eatwell, M. Milgate and P. Newman, eds., The New Palgrave: A Dictionary of Economics. First Edition. London, UK: Palgrave Macmillan, 1987.

Hannan, E.L., Kilburn, H., Racz, M., Shields, E. and Chassin, M.R. "Improving the Outcomes of Coronary Artery Bypass Surgery in New York State." Journal of the American Medical Association, Vol. 271 (1994), pp. 761-766.

Harbaugh, R. and Rasmusen. E.B. "Coarse Grades: Informing the Public by Withholding Information." Mimeo, Department of Business Economics and Public Policy, Indiana University, 2014.

Heckman, J.J. and Smith, J.A. "The Determinants of Participation in a Social Program: Evidence from a Prototypical Job Training Program.” Journal of Labor Economics. Vol. 22 (2004), pp. 243-298.

—., Heinrich, C., and Smith, J.A. "The Performance of Performance Standards." Journal of Human Resources, Vol. 37 (2002), pp. 778-811.

Holmstrom, B. "Managerial Incentive Problems: A Dynamic Perspective." Review of Economic Studies, Vol. 66 (1999), pp. 169-182.

Jacob, B.A. "Accountability, Incentives and Behavior: The Impact of High-Stakes Testing in the Chicago Public Schools." Journal of Public Economics, Vol. 89 (2005), pp. 761-796.

Jha, A.K. and Epstein, A.M. "The Predictive Accuracy of the New York State Coronary Artery Bypass Surgery Report-Card System." Health Affairs, Vol. 25 (2006), pp. 844-855.

Kane, T.J. and Staiger, D.O. "The Promise and Pitfalls of Using Imprecise School Accountability Measures." Journal of Economic Perspectives, Vol. 16 (2002), pp. 91-114.

Kolstad, J.T. "Information and Quality when Motivation is Intrinsic: Evidence from Surgeon Report Cards." American Economic Review, Vol. 103 (2013), pp. 2875-2910. 
Lang, K. "Measurement Matters: Perspectives on Education Policy from an Economist and School Board Member." Journal of Economic Perspectives, Vol. 24 (2010), pp. 167-182.

Levin, J. "Relational Incentive Contracts." American Economic Review, Vol. 93 (2003), pp. 835-857.

Lizzeri, A. "Information Revelation and Certification Intermediaries." RAND Journal of Economics, Vol. 30 (1999), pp. 214-231.

Ma, C.A. Mak H.Y. "Public Report, Price, and Quality." Journal of Economics 6 Management Strategy, Vol. 23 (2014), pp. 443-464.

—. Mak H.Y. "Information Disclosure and the Equivalence of Prospective Payments and Cost Reimbursements." Journal of Economic Behavior EG Organization, Vol. 117 (2015), pp. 439-452.

MacLeod. W.B. "Optimal Contracting with Subjective Evaluation." American Economic Review, Vol. 93 (2003), pp. 216-240.

Mailath, G.J. "Simultaneous Signaling in an Oligopoly Model." Quarterly Journal of Economics, Vol. 104 (1989), pp. 417-427.

—., Okuno-Fujiwara, M. and Postlewaite, A. "Belief-based Refinements in Signalling Games." Journal of Economic Theory, Vol. 60 (1993), pp. 241-276.

Marcin, J.P., Li, Z., Kravitz, R.L., Dai, J.J., Rocke, D.M. and Romano, P.S. "The CABG Surgery VolumeOutcome Relationship: Temporal Trends and Selection Effects in California, 1998-2004." Health Services Research, Vol. 43 (2008), pp. 174-192.

Mass-DAC "Percutaneous Coronary Intervention in the Commonwealth of Massachusetts." October 2005, October 2006, October 2007.

Matthews, S. and Postlewaite, A. "Quality Testing and Disclosure." RAND Journal of Economics, Vol. 16 (1985), pp. 328-340.

McGuire, T.G. "Physician Agency." In A.J. Culyer and J.P. Newhouse, eds., Handbook of Health Economics, Amsterdam, the Netherlands: North Holland, 2000. 
Milgrom, P.R. "Good News and Bad News: Representation Theorems and Applications." Bell Journal of Economics, Vol. 12 (1981), pp. 380-391.

—. "Is Sympathy an Economic Value? Philosophy, Economics, and the Contingent Valuation Method." In J.A. Hausman, eds., Contingent Valuation: A Critical Assessment, Amsterdam, the Netherlands: North Holland, 1993.

Resnic, F.S. and Welt, F.G.P. "The Public Health Hazards of Risk Avoidance Associated with Public Reporting of Risk-Adjusted Outcomes in Coronary Intervention." Journal of the American College of Cardiology, Vol. 53 (2009), pp. 825-830.

Rothschild, M. and Stiglitz, J. "Equilibrium in Competitive Insurance Markets: An Essay on the Economics of Imperfect Information." Quarterly Journal of Economics, Vol. 90 (1976), pp. 629-649.

Spence, M. “Job Market Signaling." Quarterly Journal of Economics, Vol. 87 (1973), pp. 355-374.

Stith, S.S. and Hirth, R.A. "The Effect of Performance Standards on Health care Provider Behavior: Evidence From Kidney Transplantation." Journal of Economics 6 Management Strategy, forthcoming.

Zhang, Y. "Are Two Report Cards Better than One? The Case of CABG Surgery and Patient Sorting." Mimeo, Department of Managerial Economics and Decision Sciences, Northwestern University, 2011. 


\section{Supplement}

This supplementary section contains two parts. The first part allows prices in the basic model in Section 4 to vary with providers' perceived service qualities. The second part uses the intuitive criterion to refine the least-cost partial pooling equilibrium in Proposition 1.

\section{Quality and price}

Section 4 studies the equilibria with a fixed service price $P$. A provider's incentive trade-off is about the masses of consumers he can serve in periods one and two. I now allow service prices to vary with consumers' perceptions about a provider's quality type. Hence, a provider's performance report can affect both his expected demand and profit margin in period two. To focus on the informational role of performance reports, I will maintain that the prices are exogenous, thereby eliminating the possibility that a provider may use price as a signalling device.

To facilitate comparison, I let the price of a $T_{i}$ provider's service be $P+\Delta\left(\widetilde{T}_{i}-\alpha G\right), 0<\Delta<1$. The consumers in period one have no information about a provider's quality type. They must infer that a provider's expected quality premium $\widetilde{T}_{i}$ is the prior $\alpha G$. Hence, the service price in period one is always equal to $P$. However, the performance reports allow the consumers in period two to update their posterior beliefs $\widetilde{T}_{i}$. A provider's service price in period two is increasing in this perception. I will continue to use $\Pi_{T}^{s}$ to denote $\omega\left(T_{i}+v^{s}\right)+(1-\omega) P$. Therefore, if a $T_{i}$ provider serves an $s$ consumer, the provider's payoff is $\Pi_{T}^{s}$ in period one, and $\Pi_{T}^{s}+(1-\omega) \Delta\left(\widetilde{T}_{i}-\alpha G\right)$ in period two.

I also incorporate price differences into the demand responses. Suppose consumers in period two believe that the quality types of Providers $i$ and $j, j \neq i$, are respectively $\widetilde{T}_{i}$ and $\widetilde{T}_{j}$. The expected mass of consumers who switch between providers is now $\tau\left[\left\{\widetilde{T}_{i}-P-\Delta\left(\widetilde{T}_{i}-\alpha G\right)\right\}-\left\{\widetilde{T}_{j}-P-\Delta\left(\widetilde{T}_{j}-\alpha G\right)\right\}\right] \widehat{q}=$ $\tau\left[(1-\Delta)\left(\widetilde{T}_{i}-\widetilde{T}_{j}\right)\right] \widehat{q}$. Because $\Delta$ is strictly less than one, a provider's expected demand is still increasing in consumers' perception about his quality type.

How would the price response change the equilibria? The service prices do not affect a provider's feasibility of gaming his performance report, hence the analysis in Section 3 follows. Now consider the incentive 


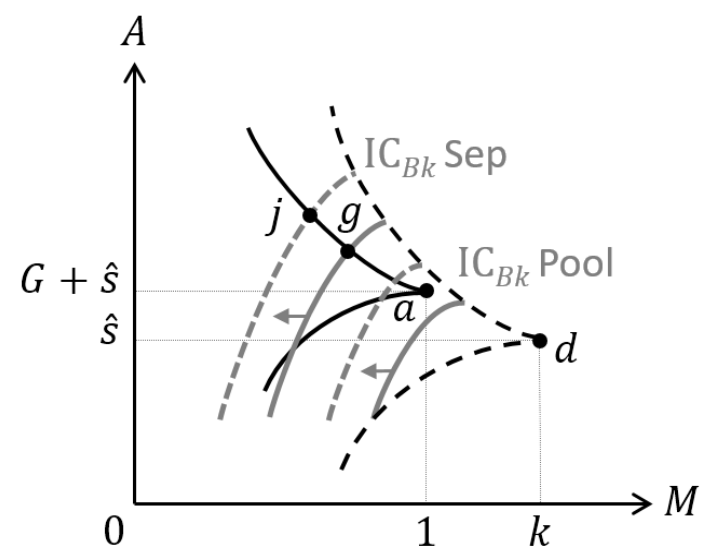

Figure 7: Price responses and incentive constraints

constraints (5), (6), (9), and (10) in Section 4. The left-hand sides of these inequalities remain unchanged, but the right-hand sides have to be expanded from $\tau \widetilde{T} \widehat{q} \widehat{\Pi}_{T}$ to

$$
\left[\tau \widetilde{T} \widehat{q} \widehat{\Pi}_{T}\right]+[\{1+\tau(1-\Delta)(\widetilde{T}-\alpha G)\} \widehat{q}(1-\omega) \Delta \widetilde{T}]-\left[\tau \Delta \widetilde{T} \widehat{q}\left\{\widehat{\Pi}_{T}+(1-\omega)(1-\Delta) \alpha G\right\}\right],
$$

where $\widetilde{T}=\frac{(1-\alpha) \beta}{\alpha(1-\beta)+(1-\alpha) \beta} G$ in a partial pooling equilibrium and $\widetilde{T}=G$ in a separating equilibrium.

The second and third squared-bracket terms in (S1) are the effects of price response on the providers' inter-period incentive trade-offs. In the second term, $\{1+\tau(1-\Delta)(\widetilde{T}-\alpha G)\} \widehat{q}$ is a provider's expected demand when his perceived quality is $\widetilde{T}$, and $(1-\omega) \Delta \widetilde{T}$ is the increase in profit margin when the provider's perceived quality increases from $B=0$ to $\widetilde{T}$. The price response also reduces the demand response when the perceived qualities of Providers $x$ and $y$ are different. This effect is captured by the third squared-bracket term in (S1). The incentive constraints in Section 4 shift parallel to the right if the sum of the two terms is positive, and vice versa. Because $\Pi_{T}^{s}=\omega\left(T_{i}+v^{s}\right)+(1-\omega) P$, the values of both terms depend on $\omega$, the weight providers put on the consumers' net payoffs.

Proposition 5 Suppose Provider $i$ 's service price is $P+\Delta\left(\widetilde{T}_{i}-\alpha G\right), 0<\Delta<1$. The price response $\Delta\left(\widetilde{T}_{i}-\alpha G\right)$ lowers $\tau_{p}^{*}$ and $\tau_{s}^{*}$, and leads to more dumping in the least-cost separating equilibrium at every $\tau, \tau_{s}^{*}<\tau<\bar{\tau}$, if

$$
0 \leq \omega<\omega^{*} \equiv \frac{\left[\frac{1}{\bar{\tau}}+(1-\Delta)\left(\frac{\alpha(1-\beta)}{\alpha(1-\beta)+(1-\alpha) \beta}-2 \alpha\right) G\right]-P}{\left[\frac{1}{\bar{\tau}}+(1-\Delta)\left(\frac{\alpha(1-\beta)}{\alpha(1-\beta)+(1-\alpha) \beta}-2 \alpha\right) G\right]+\widehat{s}-P} .
$$


A provider's weights of extrinsic and intrinsic payoffs affect his valuations between profit margin and volume in period two, hence his incentive to dump consumers in period one. By Propositions 1 and 2, the properties of the least-cost equilibria are shaped by the incentive constraints of a $B k$ provider. When $0 \leq \omega<\omega^{*}$, a $B k$ provider puts a sufficiently small weight on his consumers' payoffs, and the sum of the second and third squared-bracket terms in (S1) is positive at every $\tau$. Figure 7 illustrates how the price response alters the least-cost equilibria in this case. In the diagram, the price response shifts the $B k$ provider's two incentive constraints to the right. This allows point $a$ to be supported as a partial pooling equilibrium report, and moves the separating equilibrium report from point $g$ to point $j .50$ Because $\frac{1}{\bar{\tau}}<G$, the (identical) squared-bracket terms in (S2) are strictly positive. However, $0<\omega^{*}$ only if $P$ is smaller than its preceding squared-bracket term. ${ }^{51}$

The case where $0 \leq \omega<\omega^{*}$ is nevertheless interesting because here the price response lowers consumer welfare unambiguously. First, Figure 7 has demonstrated that providers dump (weakly) more consumers in period one. In period two, because the expected value of $\widetilde{T}-\alpha G$ is zero, the price response does not change the expected price. However, the price variations reduce the demand response. This lowers consumer welfare gain from quality information dissemination by the magnitude of $(1-\Delta)^{2} .{ }^{52}$

Proof of Proposition 5: In (8) and (11), the denominators are $\widetilde{T} \widehat{q} \widehat{\Pi}_{T}$, where $\widetilde{T}=\frac{(1-\alpha) \beta}{\alpha(1-\beta)+(1-\alpha) \beta} G$ and $\widetilde{T}=G$, respectively. Therefore, the price response must lower the values of $\tau_{p}^{*}$ and $\tau_{s}^{*}$ if the value of (S1) is larger than $\tau \widetilde{T} \widehat{q} \widehat{\Pi}_{T}$ at every $\tau$. Upon simplification, the value of (S1) is larger than $\tau \widetilde{T} \widehat{q} \widehat{\Pi}_{T}$ at a given $\tau$ if

$$
\omega<\frac{\frac{1}{\tau}+(1-\Delta)(\widetilde{T}-2 \alpha G)-P}{\frac{1}{\tau}+(1-\Delta)(\widetilde{T}-2 \alpha G)+\widehat{s}-P} .
$$

Because the right-hand side of the inequality is decreasing in $\tau$ and $\frac{(1-\alpha) \beta}{\alpha(1-\beta)+(1-\alpha) \beta}<1$, the value of (S1) is larger than $\tau \widetilde{T} \widehat{q} \widehat{\Pi}_{T}$ at every $\tau$ if condition (S2) holds.

Now let $\tau_{s}^{*} \leq \tau<\bar{\tau}$ and consider the least-cost separating equilibria. Recall $D_{G 1}^{l}\left(M_{G 1}, A_{G 1}\right)$ in (A1) is

\footnotetext{
${ }^{50}$ Provided that $\Delta$ is sufficiently small. See the proof of Proposition 5 for the necessary condition for the existence of separating equilibria.

${ }^{51}$ Note that (S2) is a sufficient condition. When $\omega^{*}<\omega$, the two constraints shift in different directions when $\omega$ is small, and both shift to the right only when $\omega$ is sufficiently close to one.

${ }^{52}$ The welfare gains from quality information dissemination in separating and partial pooling are $2 \alpha(1-\alpha)(1-$ $\Delta)^{2} \tau G^{2} \widehat{q}$ and $2 \alpha(1-\alpha)(1-\Delta)^{2} \tau G^{2} \widehat{q} \frac{\alpha(1-\beta)^{2}+(1-\alpha) \beta^{2}}{\alpha(1-\beta)+(1-\alpha) \beta}$, respectively.
} 
derived from the binding incentive constraint of a $B k$ provider. Because of the price response, $\tau G \widehat{q} \widehat{\Pi}_{B}$ in the expression has to be replaced by

$$
\left[\tau G \widehat{q} \widehat{\Pi}_{B}\right]+[\{1+\tau(1-\Delta)(G-\alpha G)\} \widehat{q}(1-\omega) \Delta G]-\left[\tau \Delta G \widehat{q}\left\{\widehat{\Pi}_{B}+(1-\omega)(1-\Delta) \alpha G\right\}\right],
$$

which has a larger value than $\tau G \widehat{q} \widehat{\Pi}_{B}$ if condition (S2) holds. Finally, upon simplification, the $G 1$ provider's incentive constraint (10) is not binding at the new $D_{G 1}^{l}$ only if the left-hand side of (A2) is bigger than

$$
\tau G \Delta \widehat{q}\left[\frac{\widehat{\Pi}_{G}}{\Pi_{G}^{l}}-\frac{\widehat{\Pi}_{B}}{\Pi_{B}^{l}}\right]+\widehat{q}(1-\omega) \Delta G(1+\tau(1-\Delta)(1-2 \alpha) G)\left[\frac{\left(\Pi_{G}^{l}-\Pi_{B}^{l}\right)+\left(\Pi_{B}^{l}-\Pi_{B}^{h}\right) \frac{G}{h-l}}{\Pi_{G}^{l} \Pi_{B}^{l}}\right] .
$$

\section{Intuitive criterion refinement}

The following proposition applies the intuitive criterion to the least-cost partial pooling equilibrium under average outcome reporting.

Proposition 6 The least-cost partial pooling equilibrium in Proposition 1 cannot be eliminated by the intuitive criterion if $h-l \leq \frac{\widehat{\Pi}_{G}}{\omega \lambda}$.

In this equilibrium, consumers in period two infer that a provider with report $(1, G+\widehat{s})$ is a good type with probability $\frac{\alpha(1-\beta)}{\alpha(1-\beta)+(1-\alpha) \beta}$. Consider a unilateral deviation with the most favorable consumers' beliefs that a provider with a different report $(\widetilde{M}, \widetilde{A})$ is a good type with probability one. A deviating provider expects to gain $\frac{(1-\alpha) \beta}{\alpha(1-\beta)+(1-\alpha) \beta} \tau G \widehat{q}$ consumers in period two. Because a $G 1$ provider dumps no consumer in the least-cost equilibrium, it is sufficient to consider $\widetilde{M}<1$. The equilibrium is eliminated by the intuitive criterion if there is at least one pair of $(\widetilde{M}, \widetilde{A})$ that is infeasible for a $B 1$ provider to obtain and

$$
\begin{aligned}
& {\left[D_{B k}^{h}(\widetilde{M}, \widetilde{A})-D_{B k}^{h}(1, G+\widehat{s})\right] \Pi_{B}^{h}+\left[D_{B k}^{l}(\widetilde{M}, \widetilde{A})-D_{B k}^{l}(1, G+\widehat{s})\right] \Pi_{B}^{l}>\frac{(1-\alpha) \beta}{\alpha(1-\beta)+(1-\alpha) \beta} \tau G \widehat{q} \widehat{\Pi}_{B}} \\
& {\left[D_{G 1}^{h}(\widetilde{M}, \widetilde{A})-D_{G 1}^{h}(1, G+\widehat{s})\right] \Pi_{G}^{h}+\left[D_{G 1}^{l}(\widetilde{M}, \widetilde{A})-D_{G 1}^{l}(1, G+\widehat{s})\right] \Pi_{G}^{l}<\frac{(1-\alpha) \beta}{\alpha(1-\beta)+(1-\alpha) \beta} \tau G \widehat{q} \widehat{\Pi}_{G}}
\end{aligned}
$$

On the left-hand sides of (S3) and (S4) are the costs of deviating from $\left(M_{p}, A_{p}\right)$ to $(\widetilde{M}, \widetilde{A})$ for $B k$ and $G 1$ providers in period one, respectively. On the right-hand sides are the gains from increasing period-two expected demands associated with deviating to $(\widetilde{M}, \widetilde{A})$. If both conditions are satisfied, only a $G 1$ provider 


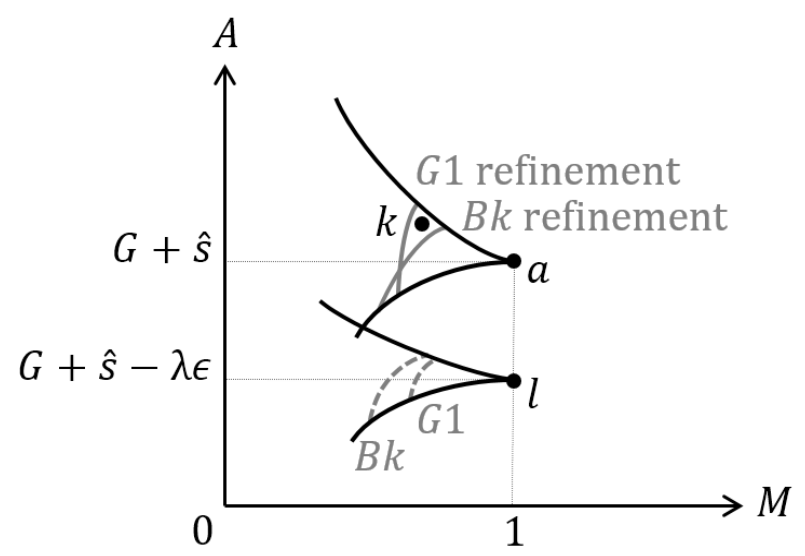

Figure 8: Refinement of partial pooling equilibrium reports

will find it profitable to unilaterally deviate from $\left(M_{p}, A_{p}\right)$ to $(\widetilde{M}, \widetilde{A})$. Thus, the period-two consumers' beliefs that a provider with the report $(\widetilde{M}, \widetilde{A})$ is a good type are justified.

The sufficient condition in Proposition $6, h-l \leq \frac{\widehat{\Pi}_{G}}{\omega \lambda}$, guarantees that the least-cost equilibrium survives the intuitive criterion. ${ }^{53}$ The inequality requires the difference between $h$ and $l$ consumers' outcomes, $h-l$, to be relatively small. Figure 8 demonstrates the intuition. First consider point $a$. This is the least-cost equilibrium report where $A_{p}=G+\widehat{s}$. Starting from this point, the upper and lower solid curves bound the combinations of $(M, A)$ that are feasible for a $G 1$ (and a $B k$ ) provider. The two solid curves on the left depict (S3) and (S4). Point $k$, which lies between the two curves, illustrates a deviation that is profitable to a $G 1$ provider only. This report eliminates the equilibrium at point $a$.

Now suppose the value of $h$ is decreased to $h-\epsilon$, where $l<h-\epsilon$. The least-cost equilibrium report is now at point $l$, where $A_{p}=G+\widehat{s}-\lambda \epsilon$. Starting from this point, the combinations of feasible $(M, A)$ have shrunk. In addition, according to (4), both (S3) and (S4) rotate clockwise, and become the two dashed curves. Because of the quality premium $G$, condition (S4) has to rotate proportionately more, and now there is no deviation that is only profitable to a $G 1$ provider. Hence, a $G 1$ provider fails to separate from a $B k$ provider when $h-l$ is small.

\footnotetext{
${ }^{53}$ Depending on the slopes of the iso-dumping cost curves, the intuitive criterion can fail to eliminate some (when $\Pi_{B}^{l}-\Pi_{B}^{h}>0$ ), or all partial pooling equilibria (when $\Pi_{B}^{l}-\Pi_{B}^{h}=0$ ).
} 
Proof of Proposition 6: A $G 1$ provider can separate from a $B k$ provider by choosing a report that (i) is feasible for both $G 1$ and $B k$ providers (and infeasible for a $B 1$ provider), and satisfies (S3), (S4); or (ii) is infeasible for a $B k$ provider (which must also be infeasible for a $B 1$ provider according to (1)). Consider the first case. Using (2), the expressions $D_{T q}^{s}(\widetilde{M}, \widetilde{A})-D_{T q}^{s}(1, G+\widehat{s}), s=h, l$, in (S3), (S4) can be rewritten as

$$
\begin{aligned}
{\left[D_{T q}^{h}(\widetilde{M}, \widetilde{A})-D_{T q}^{h}(1, G+\widehat{s})\right] } & =\frac{(T+h-G+\widehat{s})-(T+h-\widetilde{A}) \widetilde{M}}{h-l} ; \\
{\left[D_{T q}^{l}(\widetilde{M}, \widetilde{A})-D_{T q}^{l}(1, G+\widehat{s})\right] } & =\frac{(G+\widehat{s}-T-l)-(\widetilde{A}-T-l) \widetilde{M}}{h-l} .
\end{aligned}
$$

Substitute these expressions into (S3), (S4) and rearrange the terms, the two inequalities become

$$
\begin{aligned}
& \left.[(h-G+\widehat{s})-(h-\widetilde{A}) \widetilde{M}]_{\widehat{\Pi}_{B}}^{\Pi_{B}^{h}}+[(G+\widehat{s}-l)-(\widetilde{A}-l) \widetilde{M}]\right]_{\widehat{\Pi}_{B}}^{\Pi_{B}^{l}}>\left[\frac{(1-\alpha) \beta}{\alpha(1-\beta)+(1-\alpha) \beta}\right] \tau G \widehat{q}(h-l) ; \\
& {[(h+\widehat{s})-(G+h-\widetilde{A}) \widetilde{M}] \frac{\Pi_{G}^{h}}{\widehat{\Pi}_{G}}+[(\widehat{s}-l)-(\widetilde{A}-G-l) \widetilde{M}] \frac{\Pi_{G}^{l}}{\widehat{\Pi}_{G}}<\left[\frac{(1-\alpha) \beta}{\alpha(1-\beta)+(1-\alpha) \beta}\right] \tau G \widehat{q}(h-l) .}
\end{aligned}
$$

The intuitive criterion can eliminate the least-cost equilibrium only if there exists a pair of $(\widetilde{M}, \widetilde{A})$ such that the value of the left-hand side of (S5) is bigger than that of (S6), or

$$
\left(v^{l}-v^{h}\right) \frac{\omega G}{\widehat{\Pi}_{G} \widehat{\Pi}_{B}}\left[-\left\{\lambda\left(\Pi_{B}^{h}-\omega h\right)+(1-\lambda)\left(\Pi_{B}^{l}-\omega l\right)\right\}(1-\widetilde{M})-\omega(G+\widehat{s}-\widetilde{A} \widetilde{M})\right]>0
$$

where I use the identities $\Pi_{T}^{s} \equiv \omega\left(T+v^{s}\right)+(1-\omega) P$ and $\widehat{\Pi}_{T} \equiv \lambda \Pi_{T}^{h}+(1-\lambda) \Pi_{T}^{l}$ to simplify the terms.

Suppose $\omega\left(v^{l}-v^{h}\right)=0$. The left-hand side of (S7) becomes zero for any $(\widetilde{M}, \widetilde{A})$. Hence the intuitive criterion fails to eliminate the least-cost equilibrium. Now let $\omega\left(v^{l}-v^{h}\right)>0$. By condition (1), $1-\widetilde{M}=$ $D_{G 1}^{h}(\widetilde{M}, \widetilde{A})+D_{G 1}^{l}(\widetilde{M}, \widetilde{A})$ and $G+\widehat{s}-\widetilde{A} \widetilde{M}=D_{G 1}^{h}(\widetilde{M}, \widetilde{A})(G+h)+D_{G 1}^{l}(\widetilde{M}, \widetilde{A})(G+l)$. Therefore, (S7) can be rewritten as

$$
\left(v^{l}-v^{h}\right) \frac{\omega G}{\widehat{\Pi}_{G} \widetilde{\Pi}_{B}}\left[\left\{D_{G 1}^{l}(\widetilde{M}, \widetilde{A}) \lambda-D_{G 1}^{h}(\widetilde{M}, \widetilde{A})(1-\lambda)\right\} \omega(h-l)-\left\{D_{G 1}^{h}(\widetilde{M}, \widetilde{A})+D_{G 1}^{l}(\widetilde{M}, \widetilde{A})\right\} \widehat{\Pi}_{G}\right]>0
$$

This inequality fails to hold for any positive $D_{G 1}^{h}(\widetilde{M}, \widetilde{A})$ and $D_{G 1}^{l}(\widetilde{M}, \widetilde{A})$ if $\lambda \omega(h-l)<\widehat{\Pi}_{G}$. 